\title{
Genetic Nrf2 Overactivation Inhibits the Deleterious Effects Induced by Hepatocyte-Specific c-met Deletion during the Progression of NASH
}

\author{
Pierluigi Ramadori, ${ }^{1}$ Hannah Drescher, ${ }^{1}$ Stephanie Erschfeld, ${ }^{1}$ Athanassios Fragoulis, ${ }^{2}$ \\ Thomas W. Kensler, ${ }^{3}$ Christoph Jan Wruck, ${ }^{2}$ Francisco Javier Cubero, ${ }^{1,4,5}$ \\ Christian Trautwein, ${ }^{1}$ Konrad L. Streetz, ${ }^{1}$ and Daniela C. Kroy ${ }^{1}$ \\ ${ }^{1}$ Department of Medicine III, University Hospital RWTH Aachen, Aachen, Germany \\ ${ }^{2}$ Department of Anatomy and Cell Biology, University Hospital RWTH Aachen, Aachen, Germany \\ ${ }^{3}$ Department of Pharmacology \& Chemical Biology, School of Medicine, University of Pittsburgh, Pittsburgh, PA, USA \\ ${ }^{4}$ Department of Immunology, Complutense University School of Medicine, Madrid, Spain \\ ${ }^{5} 12$ de Octubre Health Research Institute (imas12), Madrid, Spain
}

Correspondence should be addressed to Daniela C. Kroy; dkroy@ukaachen.de

Received 12 December 2016; Accepted 22 March 2017; Published 6 June 2017

Academic Editor: M. Yvonne Alexander

Copyright (c) 2017 Pierluigi Ramadori et al. This is an open access article distributed under the Creative Commons Attribution License, which permits unrestricted use, distribution, and reproduction in any medium, provided the original work is properly cited.

\begin{abstract}
We have recently shown that hepatocyte-specific c-met deficiency accelerates the progression of nonalcoholic steatohepatitis in experimental murine models resulting in augmented production of reactive oxygen species and accelerated development of fibrosis. The aim of this study focuses on the elucidation of the underlying cellular mechanisms driven by Nrf2 overactivation in hepatocytes lacking c-met receptor characterized by a severe unbalance between pro-oxidant and antioxidant functions. Control mice $\left(c-\right.$ met $\left.^{\mathrm{fx} / \mathrm{fx}}\right)$, single c-met knockouts (c-met ${ }^{\Delta \text { hepa }}$ ), and double c-met/Keap1 knockouts (met/Keap $1^{\Delta \text { hepa }}$ ) were then fed a chow or a methionine-choline-deficient (MCD) diet, respectively, for 4 weeks to reproduce the features of nonalcoholic steatohepatitis. Upon MCD feeding, met/Keap1 $1^{\Delta h e p a}$ mice displayed increased liver mass albeit decreased triglyceride accumulation. The marked increase of oxidative stress observed in c-met ${ }^{\Delta \text { hepa }}$ was restored in the double mutants as assessed by 4-HNE immunostaining and by the expression of genes responsible for the generation of free radicals. Moreover, double knockout mice presented a reduced amount of liver-infiltrating cells and the exacerbation of fibrosis progression observed in $c$-met ${ }^{\Delta \text { hepa }}$ livers was significantly inhibited in met/Keap $1^{\Delta \text { hepa }}$. Therefore, genetic activation of the antioxidant transcription factor Nrf2 improves liver damage and repair in hepatocyte-specific c-met-deficient mice mainly through restoring a balance in the cellular redox homeostasis.
\end{abstract}

\section{Introduction}

Formation of reactive oxygen species (ROS) has been considered classically a pathophysiological phenomenon critically involved in the progression from simple hepatic steatosis to steatohepatitis. Upon triglyceride accumulation, cellular compartments responsible for lipid catabolism such as mitochondria and lysosomes increase their activity with a consequent generation of free radicals that trigger molecular signals leading to cell death and release of proinflammatory mediators. In this context, the use of antioxidant buffering against the generation of ROS has been shown to partially reduce the progression of nonalcoholic steatohepatitis. A growing body of evidence indicates the HGF (hepatocyte growth factor)/c-met axis as a molecular pathway linked to the control of the cellular redox homeostasis. However, data concerning the consequences of HGF stimulation on the cellular generation of free radicals are still quite controversial. Whereas in primary cell lines and tissues such as cardiomyocytes [1] and neurons [2], stimulation with HGF was shown 
to dampen ROS production and to reduce oxidative stressdependent apoptosis; in cancer cell lines [3] and other in vitro conditions [4], HGF treatment resulted in augmented cell motility accompanied by increased ROS production. Our group and others recently showed that disruption of c-met functionality aggravates the onset of NASH through the impairment of mechanisms regulating cell sensitivity to lipotoxicity, ROS production, and cell proliferation $[5,6]$. In particular, data emerging from genomic array analysis clearly indicated an aberrant regulation of a pattern of genes responsible for increased pro-oxidant environment, amongst them the transcription factor Nrf2 (nuclear factor erythroid-derived 2-like 2) [5].

Under conditions of oxidative or electrophilic stress, Nrf2 degradation is inhibited through oxidant-dependent modifications of specific cysteine residues within Keap1, a protein that under quiescent conditions facilitates the marking of Nrf2 for degradation via the proteasome [7]. It is now well established that stabilization and activation of Nrf2 through pharmacological or genetic targeting improves cellular redox homeostasis and survival through transcriptional upregulation of antioxidant and detoxifying genes [8]. In line with these studies, we recently showed that activation of Nrf2 in hepatocytes afforded by genetic deletion of Keap1 was able to reduce triglycerides accumulation and ROS generation in mice subjected to experimental models of NASH [9]. This former observation leads to the generation of a double knockout mouse lacking simultaneously the receptor c-met and Keap1 specifically in hepatocytes to investigate the effects of Nrf2 overexpression in cells displaying an impaired control of the redox functions. In fact, the purpose of this study pointed towards uncovering a bridge between the HGF/c-met axis and the Keap1/Nrf2 system in the context of metabolic liver disturbances (schematic overview in Suppl. Fig. 4 available online at https://doi.org/ $10.1155 / 2017 / 3420286$ ). The results emerging indicated that overexpression of $\mathrm{Nrf} 2$ was able to suppress the levels of liver steatosis and fibrosis in c-met-deficient hepatocytes to the level of the control group, with a drastic reduction of triglyceride (TG) accumulation and ROS production. Considering that preliminary clinical data indicate a reduction of c-met expression in patients diagnosed with NASH, this study provides further evidence for strategies for therapeutic interventions in this field.

\section{Materials and Methods}

2.1. Animals and Experimental Model. Hepatocyte-specific Keap1 knockout mice were generated by breeding Keap1floxed mice with albumin-Cre (Alb-Cre) C57BL/6 transgenic mice as previously described [10]. Similarly, floxed wild type $\left(\mathrm{c}-\mathrm{met}^{\mathrm{fx} / \mathrm{fx}}\right)$ and hepatocyte-specific conditional c-met knockout $\left(\mathrm{c}-\mathrm{met}^{\Delta \text { hepa }}\right)$ mice were generated under control of a postnatal activated albumin promoter $(\mathrm{C} 57 \mathrm{BL} / 6)$, as indicated elsewhere [11]. These two strains were then crossed to generate double mutant $\mathrm{c}$-met/Keap1 (met/Keap $1^{\Delta \text { hepa }}$ ) harboring the same genetic background. Male age-matched wild type $\left(\mathrm{c}-\mathrm{met}^{\mathrm{f} / \mathrm{fx}}\right)$ and littermate hepatocyte-specific conditional c-met-knockout $\left(c-\right.$ met $\left.^{\Delta \text { hepa }}\right)$ were then cohoused with genetically derived met/Keap1 $1^{\Delta \text { hepa }}$ in 12-hour light/ dark cycle and allowed to free food and water. At the age of 8-10 weeks, the animals were split in three groups $(N=5)$ and fed a chow and MCD diet (E15652-94, ssniff Spezialdiäten $\mathrm{GmbH}$ ), respectively, for a period of 4 weeks. Food intake and body weights were measured weekly, and all animals consumed similar amounts of diets. At the end of the indicated time point, blood and liver samples were collected, fixed in formalin, and cryopreserved for biochemical and histological analyses. All animal experiments were carried out in accordance with the regulations of the German legal requirements on laboratory animal care (LANUV).

2.2. Histological and Morphological Analyses. After explant, liver tissues were fixed in formaldehyde-buffered solution for $24 \mathrm{~h}$ and then embedded in paraffin. Blocks containing preserved hepatic tissues were then cut into $5 \mu \mathrm{m}$ sections and stained with hematoxylin and eosin ( $\mathrm{H} \& \mathrm{E})$ for microscopic examination and morphological analyses. Similarly, $8 \mu \mathrm{m}$ thin liver sections were stained, upon deparaffinization, with a Sirius red/picric acid (Sigma-Aldrich) solution for $1 \mathrm{~h}$ as previously described [9]. Upon dehydration and mounting, photomicrographs of stained sections were randomly taken in a $200 \mathrm{x}$ magnification and positive areas were quantified using the open source software ImageJ.

2.3. Serum and Liver Biochemical Measurements. Blood samples were collected and centrifuged in heparin-embedded tubes, and serum transaminase levels (ALT/AST) were measured according to the standard procedures of the Central Laboratory Facility of the University Hospital RWTH of Aachen as index of liver damage. For the evaluation of intrahepatic triglyceride content, liver samples were homogenized in a specific Tris buffer (10 mM Tris, $2 \mathrm{mM}$ EDTA, $0.25 \mathrm{M}$ sucrose, and $\mathrm{pH}$ 7.5) and successively processed according to the manufacturer's instructions of a commercial colorimetric kit (10724600, Human Diagnostics). For biochemical quantification of hepatic collagen deposition, $50 \mathrm{mg}$ of liver samples were homogenized in $1 \mathrm{ml}$ of $6 \mathrm{~N} \mathrm{HCl}$ solution and incubated o.n. at $110^{\circ} \mathrm{C}$. Homogenates were then treated with a chloramine- $\mathrm{T}$ solution and incubated with Ehrlich's reagent to measure hydroxyproline content by biochemical photometric assay as indicated in previous publication [12].

2.4. RNA Isolation and $q P C R$ Analysis. Total RNA from hepatic tissue was isolated with peqGold RNAPure solution (30-1020, PeqLab, VWR, Germany) according to the manufacturer instructions. An amount of $500 \mathrm{ng}$ of isolated RNA was transcribed into cDNA using the Omniscript Reverse Transcription kit (205111, Qiagen). Quantitative qPCR was performed by using a Real-time PCR System 7300 (Applied Biosystems) and Fast SYBR Green Master Mix qPCR (Thermo Fisher Scientific). Relative gene expression was calculated using the $2^{-\Delta \Delta \mathrm{Ct}}$ quantification formula normalizing each gene with the expression of the housekeeping gene $18 \mathrm{~S}$ (ribosomal subunit). The primers used in this study are reported in Suppl. Table 1.

2.5. Western Blot Analyses. Total hepatic homogenates were performed by lysing liver samples with an Ultra-Turrax 
homogenizer in Tris/HCl-lysis buffer containing inhibitors of proteases and phosphatases as described before [9]. Fifty to eighty micrograms of total lysates were denatured in Laemmli sample buffer and separated in $10 \%$ and $12 \%$ SDS-PAGE gel. Upon electrophoresis and transfer blotting, Ponceau-Red staining was used to verify transfer efficiency and equal protein loading. Membranes were then blocked in blocking buffer and incubated overnight at $4^{\circ} \mathrm{C}$ with primary antibodies (Suppl. Table 2). Therefore, secondary antibodies were incubated on the membranes for one hour at room temperature. The following secondary antibodies have been used in this study: HRP-linked anti-rabbit immunoglobulin G (7074, Cell Signaling) and HRP-linked antimouse immunoglobulin G (sc-2005, Santa Cruz). Enhanced chemiluminescence (ECL) method was used to detect protein bands and the software ImageJ was employed for densitometric analysis of band intensity.

2.6. FACS Analysis of Myeloid and Lymphoid Hepatic Infiltrates. A nonparenchymal cell fraction from whole liver extracts was isolated upon collagenase and mechanical digestion followed by Percoll (GE Healthcare Life Sciences) gradient centrifugation as previously described [5]. In parallel, blood samples were collected in EDTA-containing tubes and treated with red blood cell lysis buffer (PharmLyse, BD Biosciences, Germany). Upon removal of red bodies and centrifugation, immune cells were incubated with fluorochrome-conjugated antibodies and characterized according to two different panels, a myeloid panel: CD45BV510 (103138, BioLegend), 7AAD-PE-Cy5-YG (420404, BioLegend), CD11b-BV711 (101242, BioLegend), F4/80APC (17-4801-82, eBiosciences), MHC2-Alexa700 (107622, BioLegend), CD11c-PE-Cy7 (25-0114-81, eBiosciences), and Ly6G-FITC (551460, BD Pharmingen) and a lymphoid panel: CD45-BV510 (103138, BioLegend), 7AAD-PE-Cy5-YG (420404, BioLegend), CD3-PE-Cy7 (25-0031-82, eBiosciences), CD4-FITC (11-0041-85, eBiosciences), CD8-PerCpCy5.5 (126610, BioLegend), and NK1.1-BV711 (108745, BioLegend). Labeled cells were then subjected to flow cytometry using a BD Canto II (BD Biosciences) and relative cell numbers were analyzed using FlowJo software (Tree Star).

2.7. Immunohistochemistry and TUNEL Assay. For 4-HNE immunostaining, paraffin-embedded sections were used. Upon antigen retrieval in sodium citrate buffer, endogenous peroxidases were inhibited through incubation with 3\% $\mathrm{H}_{2} \mathrm{O}_{2}$ in PBS buffer for 10 minutes. Blocking was performed by incubating the sections with $5 \%$ goat serum in PBS buffer for 1 hour. Sections were then incubated with primary antibody in blocking solution overnight at $4^{\circ} \mathrm{C}$ and successively 1 hour at room temperature with secondary antibody (anti-mouse biotinylated). Antigen was visualized using a peroxidase substrate DAB kit (di-amino benzidine) (DAKO). For immunofluorescence staining, hepatic $5 \mu \mathrm{m}$ cryosections were fixed in $4 \%$ paraformaldehyde-buffered solution. Blocking was performed by incubating the sections with $0.2 \%$ BSA in PBS buffer for 5 minutes. Then sections were incubated with primary antibody for $1 \mathrm{~h}$ at RT in PBS buffer containing $1 \%$ mouse serum. After washing and further blocking, slides were incubated with secondary antibody for one hour at RT. DAPI (4',6-diamidino-2-phenylindole) was used to visualize cell nuclei. The secondary antibody used in this section is anti-rat Alexa Fluor 594-conjugated antibody (Molecular Probes/Invitrogen). Primary antibodies are indicated in Suppl. Table 2.

For the detection of apoptotic cells, a TUNEL (terminal deoxynucleotidyl transferase dUTP nick end labeling) assay was performed by using the in situ cell death detection kit (Fluorescein, 11684795910, Roche). Analysis of quantification of positive cells was performed by using the open source software ImageJ.

2.8. Statistical Analysis. All results are expressed as mean $\pm \mathrm{SE}$ and represent data from 5 animals per group. All significant $p$ values were measured by one-way ANOVA test, followed by Bonferrroni's posttest for the comparison between groups. A value of $p<0.05$ was considered significant $\left({ }^{*} p<0.05\right.$, $\left.{ }^{* *} p<0.01\right)$.

\section{Results}

3.1. Nrf2 Overexpression in Hepatocytes Lacking c-met Receptor Results in Reduced Triglycerides Accumulation upon MCD Feeding. As previously described, deletion of the exon 15 in c-met ${ }^{\Delta \text { hepa }}$ mice operated by the crerecombinase under control of the albumin promoter resulted in a defective intracellular activation of the receptor as indicated by impaired phosphorylation of specific tyrosine residues (data not shown). The effective deletion of c-met exon 15 was confirmed by reverse transcriptase-PCR analysis (Figure 1(a), upper graph). As expected, selective hepatic deletion of Keap1 resulted in increased protein levels of the transcription factor Nrf2 (Figure 1(a), lower graph). This stabilization correlated with augmented transcriptional activity as assessed by expression of a well-known target gene Nqo1 (Suppl. Fig. 1a). Displaying no differences of total body weight, hepatocyte c-met deletion resulted in a slight reduction of the hepatic mass. Further, deletion of Keap 1 induced a moderate but significant increase of liver weight as compared to control mice, under normal chow feeding, only subtly depending on changes of the cell proliferation rate (Suppl. Fig. 1b). However, microscopically, we could not detect any obvious alteration as emerged from histological analysis of hematoxylin and eosin (H\&E) staining (Figure 1(b), left panels). After 4 weeks of MCD feeding, met/Keap ${ }^{\text {Shepa }}$ still displayed a significant increase of the liver mass without significant alterations of body weight that progressively declined in all groups (Figures $1(\mathrm{c})$ and $1(\mathrm{~d})$ ). Interestingly, double knockouts showed decreased accumulation of lipid droplets and a lower grade of steatosis as confirmed by histological analyses of H\&E staining (Figure 1(b), right panels). Biochemical analyses revealed that met/Keap1 ${ }^{\Delta \text { hepa }}$ accumulated about $50 \%$ less TG in the liver as compared to other experimental groups (Figure 1(e)). Accordingly, gene expression of the lipid droplet associated protein, Plin2, showed an absolute increase of steatohepatitis progression in c-met ${ }^{\Delta \text { hepa }}$ mice. In contrast, the same gene was significantly downregulated 


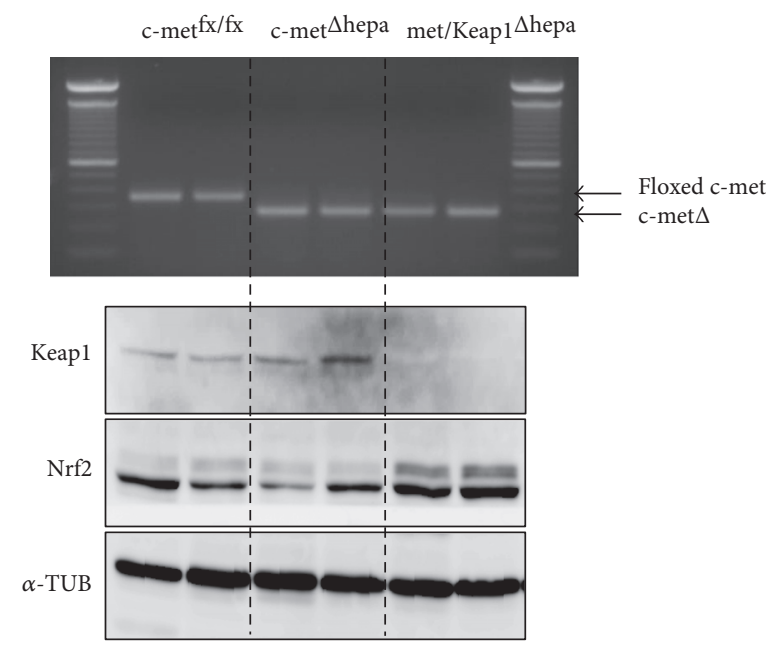

(a)

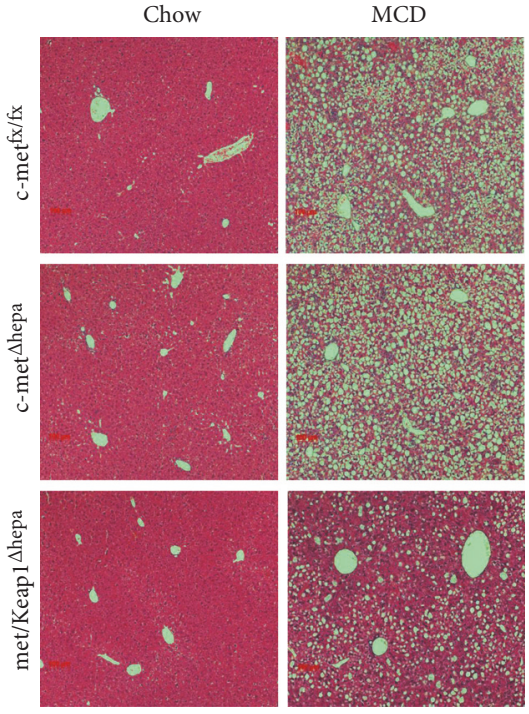

(b)

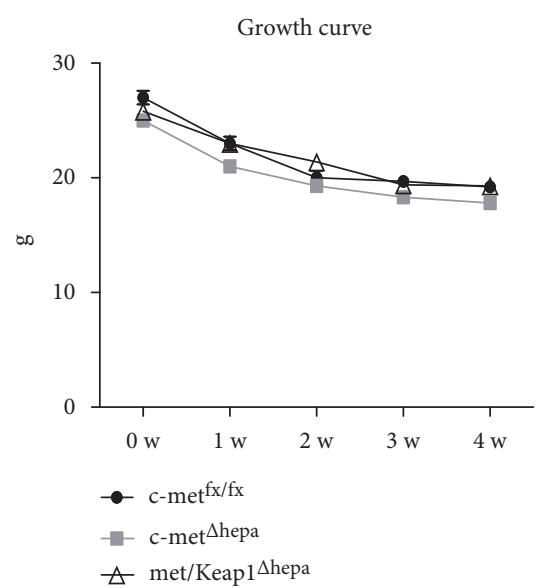

(c)

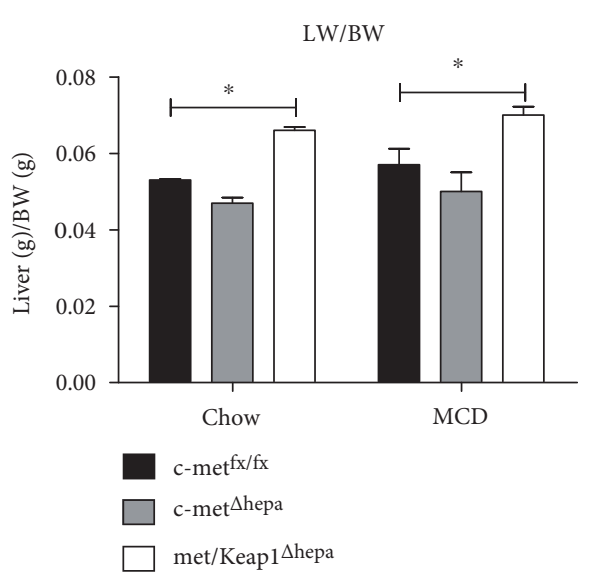

(d)

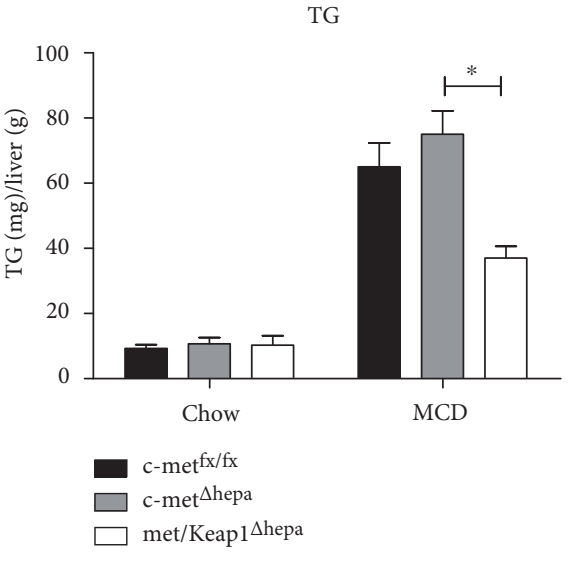

(e)

FIGURE 1: Genotyping of c-met gene performed on cDNA transcribed from hepatic RNA showing amplification of the floxed region of the gene with and without cre-recombinase activation ((a), upper panel). Western blot panel for Keap1 and Nrf2 protein expression analysis ((a), lower panels). Representative pictures of hematoxylin and eosin (H\&E) staining on liver section of c-met ${ }^{\mathrm{fx} / \mathrm{fx}}, \mathrm{c}-\mathrm{met}{ }^{\Delta \mathrm{hepa}}$, and $\mathrm{met} /$ Keap $1^{\Delta \text { hepa }}$ mice after 4 weeks chow (left panels) and MCD (right panels) diet (b). Weekly growth curve of $\mathrm{c}-\mathrm{met}^{\mathrm{fx} / \mathrm{fx}}$, c-met ${ }^{\Delta \text { hepa }}$, and met/Keap $1^{\Delta \text { hepa }}$ mice during a 4 -week course of MCD treatment. ${ }^{*}$ Data are expressed as mean \pm SE, Student's $t$-test with $p<0.05(N=5)$ (c). Liver body weight ratio (d) and intrahepatic triglyceride content measurement (e) after 4-week chow and MCD diet feeding. ${ }^{*}$ Data are expressed as mean $\pm \mathrm{SE}$, ANOVA test with $p<0.05(N=5)$.

in $\mathrm{met} / \mathrm{Keap1}^{\Delta \text { hepa }}$ hepatocytes as compared with the single mutants (Figure 2(a)). Furthermore, double knockout mice displayed increased hepatic expression and phosphorylation of AMPK and augmented expression of the transcription factor PGC- $1 \alpha$ compared to the other experimental groups (Figures 2(b), 2(c), and 2(d)), indicating enhanced fatty acid oxidation and mitochondrial biogenesis. These results confirmed that $\mathrm{Nrf} 2$ overactivation is sufficient to enhance hepatic lipid catabolism and mitochondrial functionality as we recently illustrated in detail elsewhere [9].

3.2. Nrf2 Overexpression Dampens the Exacerbation of Oxidative Stress Production in Hepatocytes Lacking c-met Receptor upon MCD Feeding. Whereas under chow diet, oxidative stress was barely detectable, and after 4 weeks of MCD administration, hepatocyte-specific c-met deletion resulted in increased production of oxidative stress compared to wild type, as evidenced by immunostaining analysis of 4-HNE, a bioproduct of lipid peroxidation (Figures 3(a), 3(b), 3(c), $3(\mathrm{~d})$, and 3(e)). In contrast, overactivation of Nrf2 induced by Keap 1 deletion resulted in a strong decrease of 4 -HNEpositive cells at levels even lower than the control group $\left(\mathrm{c}-\mathrm{met}^{\mathrm{fx} / \mathrm{fx}}\right.$ ) (Figure 3(f)). Of note, histological analyses indicated that met/Keap $1^{\Delta \text { hepa }}$ livers not only showed a decreased number of 4 -HNE positive cells but the intensity of the signal was also lower compared to $\mathrm{c}-\mathrm{met}^{\mathrm{fx} / \mathrm{fx}}$ and single c-met $^{\text {thepa }}$ (Figures 3(d), 3(e), and 3(f)). More importantly, as also observed in our previous microarray analyses [5], c-met deletion resulted in derepression of pro-oxidant enzymes directly responsible for the generation of cellular 


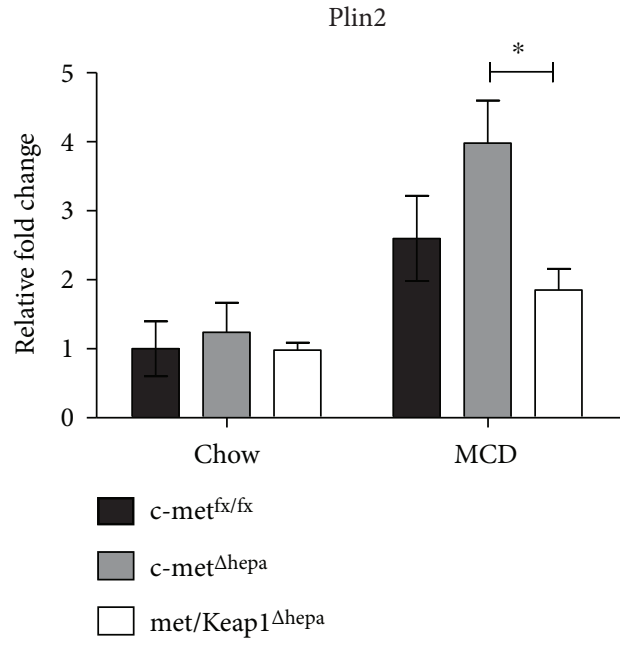

(a)

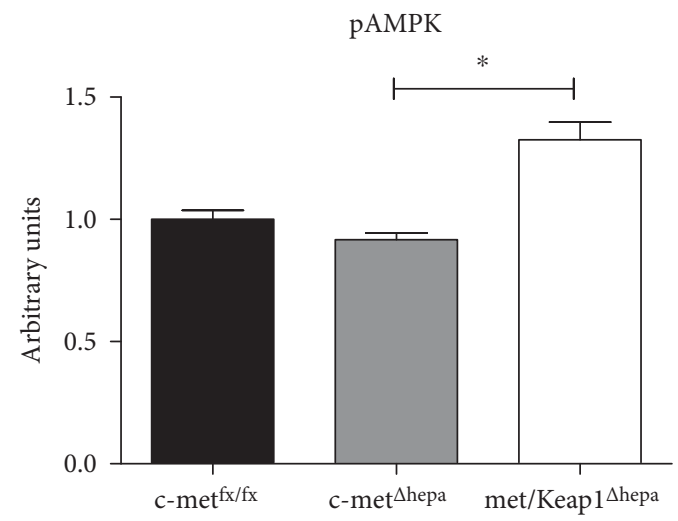

(c)

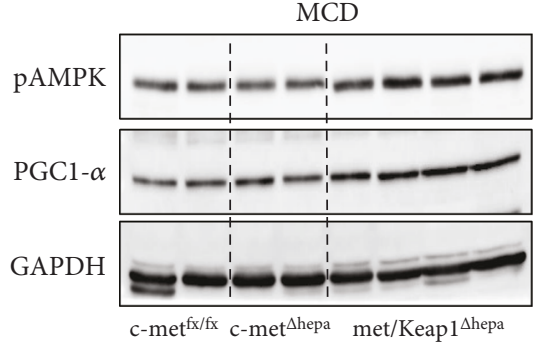

(b)

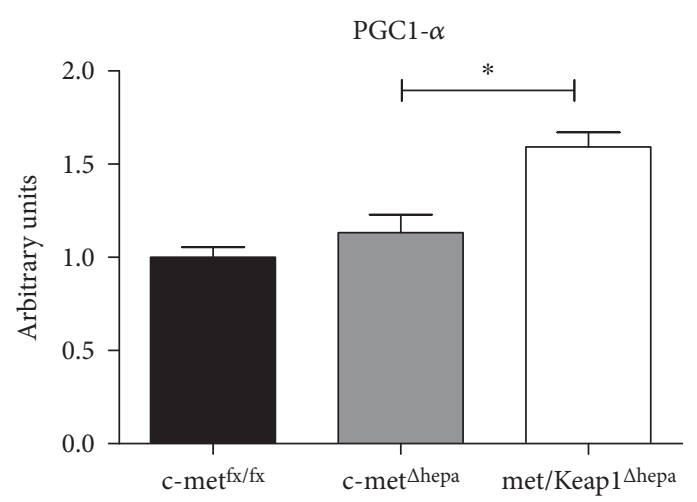

(d)

Figure 2: Analysis of hepatic gene expression of proteins involved in lipid droplet formation (a). Hepatic protein expression levels from Western blot (b) and densitometric analysis of band intensities ( $c$ and d) performed on total liver lysates of mice after $4 \mathrm{w}$ MCD administration. ${ }^{*}$ Data are expressed as mean \pm SE, ANOVA test with $p<0.05(N=5)$.

ROS, such as Cyp2e1, Cyp4a10, and the NADPH oxidase NOX2 (Figures 4(a), 4(b), and 4(c)). Thus, hepatic induction of these genes as well as the protein expression (Figures $4(\mathrm{~g})$ and $4(\mathrm{~h})$ ) was dramatically blunted by overexpression of Nrf2 in met/Keap1 ${ }^{\Delta \text { hepa }}$ mice. These results seem to offer further evidence for a role of HGF/c-met signaling in the preservation of the cellular redox balance. Furthermore, Nrf2 activation resulted in upregulation of gene expression of enzymes involved in $\mathrm{H}_{2} \mathrm{O}_{2}$ and free radical scavenging, such as catalase, thioredoxin- 1 , and the pentose phosphate pathway intermediate enzyme 6-phosphogluconate dehydrogenase (PGD) (Figures 4(d), 4(e), and 4(f)).

\subsection{Nrf2 Overexpression Drastically Reduces the Number of} Apoptotic Cells in Hepatocytes Lacking c-met Receptor upon $M C D$ Feeding. In association with increased ROS production, TUNEL assay revealed that the number of apoptotic hepatocytes in c-met ${ }^{\text {thepa }}$ livers was augmented as compared to the other experimental groups (Figures 5(a), 5(b), 5(c), and 5(d)), as already observed in other experimental situations. Concomitant Keap1 deletion in c-met ${ }^{\Delta h e p a}$ hepatocytes significantly turned down programmed cell death to levels comparable with the control group (Figure 5(d)) although no significant differences in the serum transaminase levels were detected (data not shown). As previously observed, Nrf2 activation correlated with a dramatic increase of the antiapoptotic protein Bcl-2 (Figure 5(e)). Surprisingly, phosphorylation levels of Akt were reduced in c-met ${ }^{\Delta h e p a}$ as well as met/ Keap $1^{\text {shepa }}$ hepatocytes, ruling out an involvement of this pathway in Nrf2-mediated cell survival (Figure 5(e)). Interestingly, met/Keap $1^{\Delta \text { hepa }}$ livers showed a significant increase of phosphorylation of the JNKs-specifically JNK1-kinases (Figures 5(e) and 5(f)) with no evident differences for other MAPK kinases such as ERK1/2 (data not shown).

3.4. Hepatic Nrf2 Overexpression in Livers Lacking c-met Receptor Decreases the Influx of Infiltrating Inflammatory Cells upon MCD Feeding. FACS analysis performed on liver lysates after 4 weeks of MCD treatment revealed that met/ Keap $1^{\Delta \text { hepa }}$ accumulate less proinflammatory neutrophils compared to the other experimental groups, as indicated by the number of $\mathrm{Ly}_{6 \mathrm{G}}^{+}$cells (Figures 6(a), 6(b), 6(c), and $6(d))$. Interestingly, the number of circulating neutrophils was also significantly reduced in these animals (Suppl. Fig. 


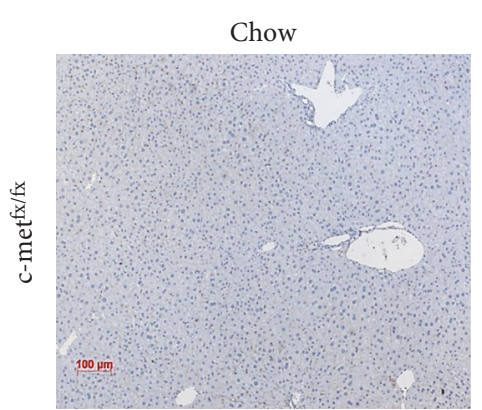

(a)

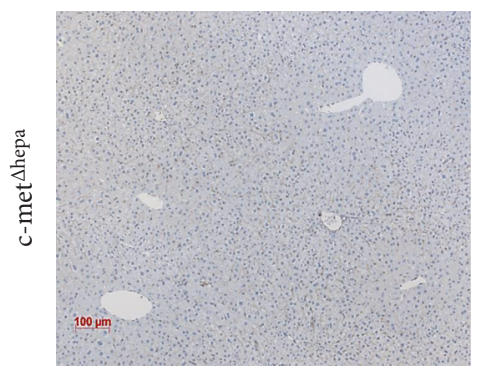

(b)

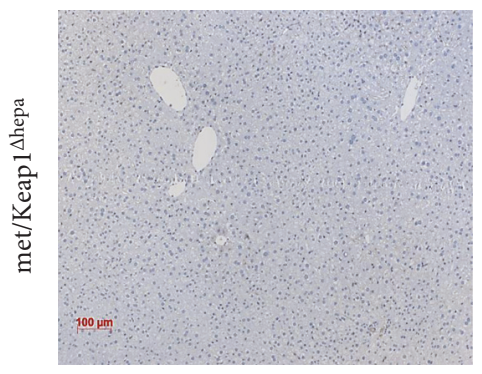

(c)

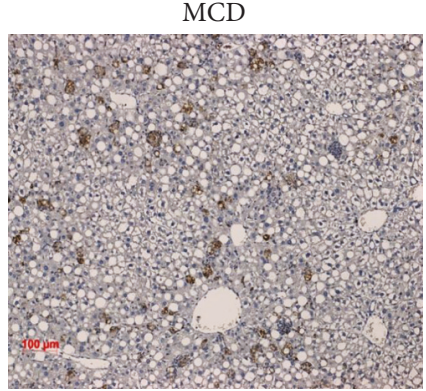

(d)

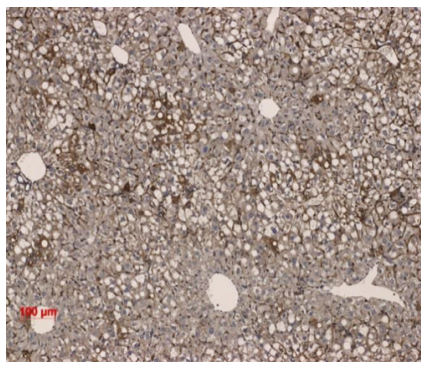

(e)

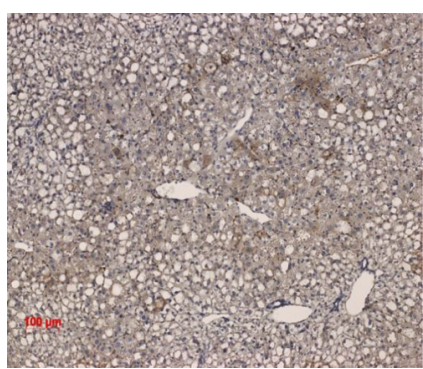

(f)

FIGURE 3: Immunohistochemistry staining for oxidative stress marker 4-HNE on liver sections of c-met ${ }^{\mathrm{fx} / \mathrm{fx}}$, c-met ${ }^{\Delta \mathrm{hepa}}$, and met/Keap ${ }^{\Delta \mathrm{hepa}}$ mice after 4-week chow (a, b, c) and MCD (d, e, f) diet administration, respectively.

3b). Similarly, the number of activated macrophages, measured as $\mathrm{F} 4 / 80^{+} / \mathrm{CD}_{11 \mathrm{~b}}{ }^{+}$cells, was dramatically reduced in the livers of met/Keap $1^{\Delta \text { hepa }}$ compared to the other groups. In line with these findings, hepatic gene expression of proinflammatory cytokines, Ccl-2 and TNF- $\alpha$, was dramatically downregulated in met/Keap1 ${ }^{\Delta \text { hepa }}$ mice (Suppl. Fig. 1c and data not shown). These results were confirmed by immunofluorescence analyses of CD11b-positive cells (Figures 7(a), 7(b), and 7(c)) and F4/ 80-positive macrophages (Figures $7(\mathrm{~d}), 7(\mathrm{e})$, and $7(\mathrm{f})$ ) supported by morphometric quantifications (Figures $7(\mathrm{~g})$ and 7(h)). Importantly, these histological pictures pointed out the dramatic reduction of the number of inflammatory cell clusters in met/Keap $1^{\Delta \text { hepa }}$ livers after MCD administration. Surprisingly, met/Keap $1^{\Delta \text { hepa }}$ mice showed a significant increase of $\mathrm{CD}^{+}$lymphocytes infiltrating into the liver whereas no changes in the $\mathrm{CD}^{+}$lymphocytes number were observed (Suppl. Fig. 2 and Suppl. Fig. 3a).

3.5. Nrf2 Overexpression in Hepatocytes Attenuates the Enhanced Development of Fibrosis Resulting from Hepatocyte-Specific c-met Deletion upon MCD Feeding. As also described in our previous report [5], c-met deletion in hepatocytes accelerates appearance and progression of liver fibrosis in several models of chronic liver injury as well as by feeding a MCD diet. Single c-met knockout livers displayed an increased deposition of collagen fibers as observed in the Sirius red staining (Figures 8(a), 8(b), and 8(c)) and confirmed by related morphometric analysis (Figure $8(\mathrm{~d})$ ). This observation was further confirmed by biochemical measurement of intrahepatic hydroxyproline content indicating the highest collagen accumulation in c-met ${ }^{\Delta \text { hepa }}$ livers (Figure $8(\mathrm{e})$ ). Interestingly, fibrosis was strongly inhibited in the double mutants where Nrf2 was overactivated. Thus, Sirius red staining and hydroxyproline content were suppressed to the levels of the control group (Figures 8(a), 8(b), 8(c), 8(d), and 8(e)). Concordantly, deletion of Keap1 in hepatocytes lacking c-met also restored the hepatic expression of profibrotic mediators as Col1A1 and TGF- $\beta 1$ to control levels (Figures $8(\mathrm{f})$ and $8(\mathrm{~g})$ ).

\section{Discussion}

The generation of reactive oxidative species associated with mitochondrial alterations and the activation of pro-oxidant enzymes still represents an unsolved issue in the context of 


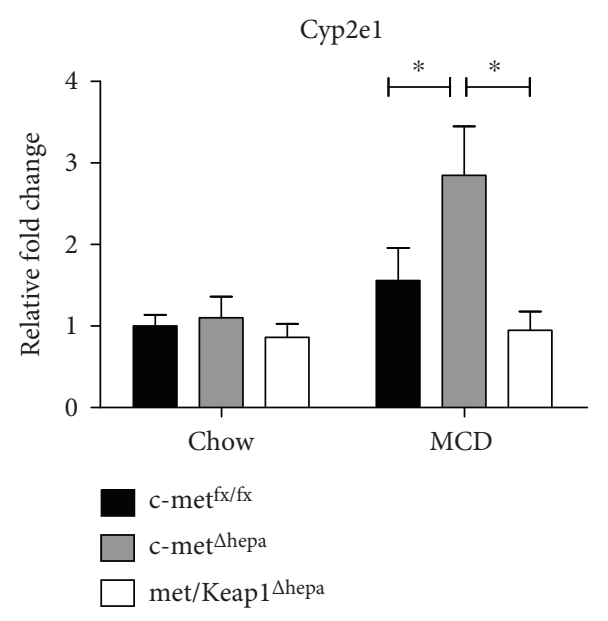

(a)

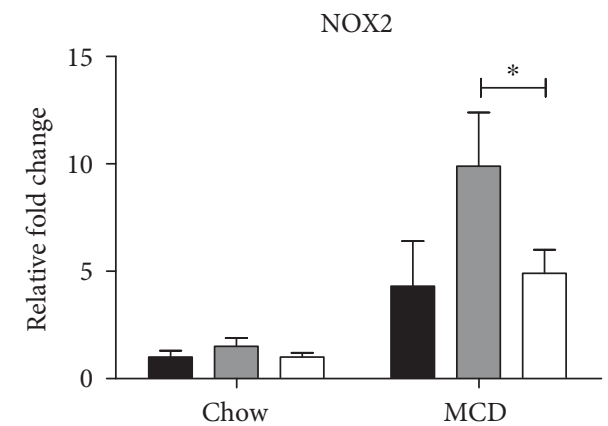

(c)

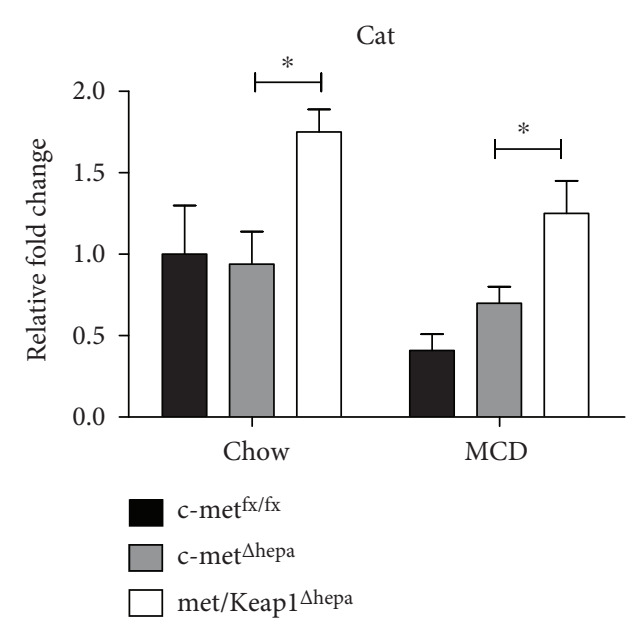

(e)

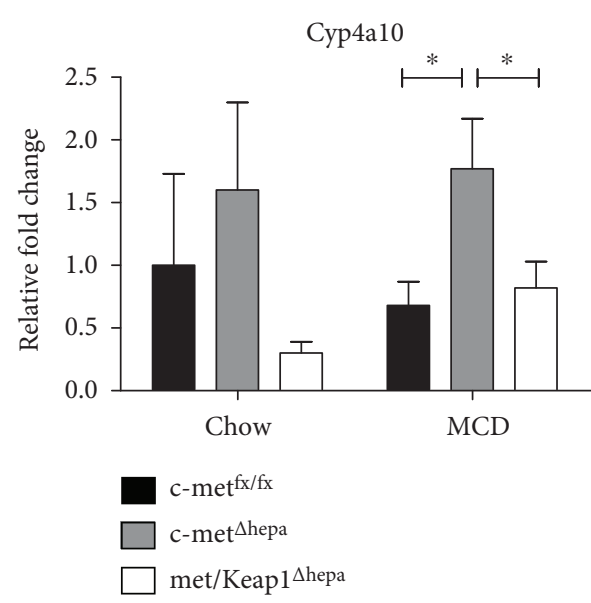

(b)
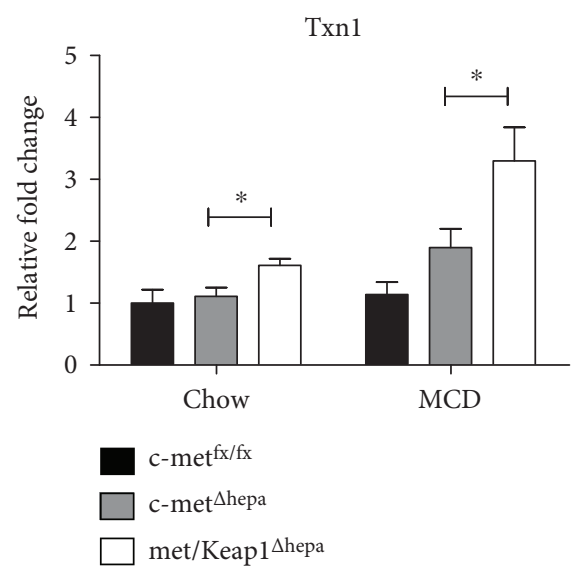

(d)

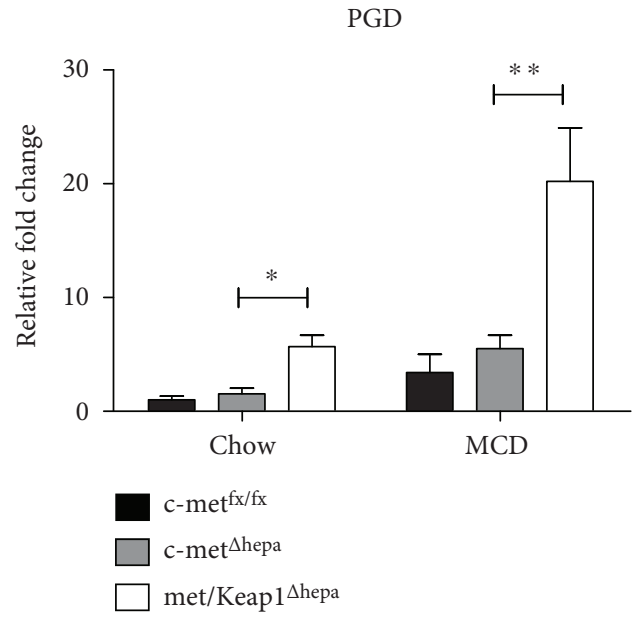

(f)

Figure 4: Continued. 


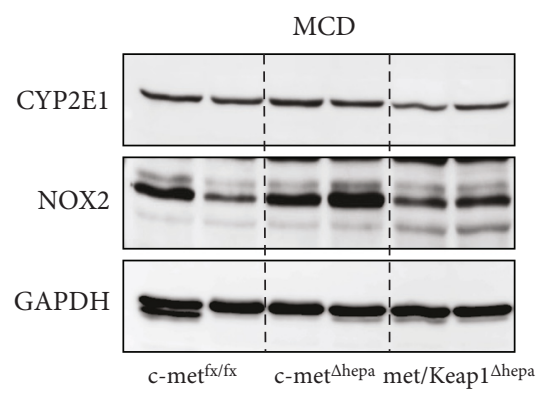

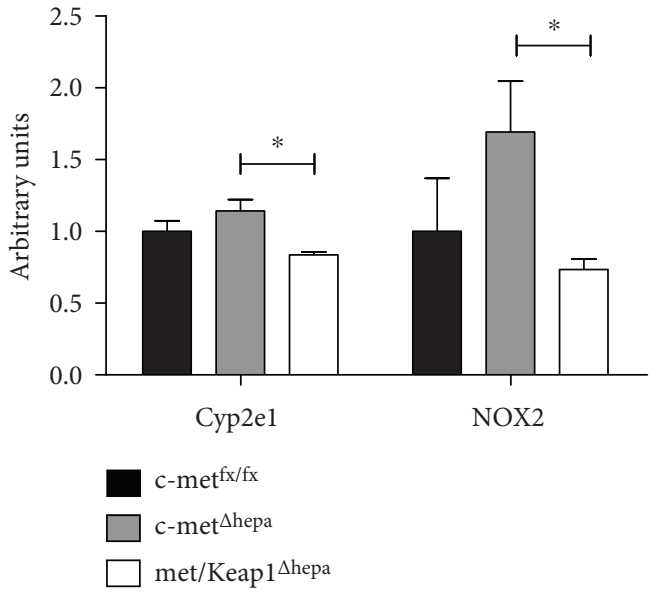

(h)

FIgURE 4: Analysis of hepatic gene expression of proteins involved ROS production (a, b, c) and of proteins responsible for the antioxidant cell defense $(\mathrm{d}, \mathrm{e})$. (f) ${ }^{* *} p$ values $<0.01$. ${ }^{*}$ Data are expressed as mean $\pm \mathrm{SE}$, ANOVA test with $p<0.05(N=5)$. Hepatic protein expression levels of Cyp2e1 and NOX2 from Western blot performed on total liver lysates of mice after $4 \mathrm{w}$ MCD administration (g) with relative densitometric analysis (h).

nonalcoholic steatohepatitis in which it seems to play a pivotal role in the exacerbation of liver injury, inflammation, and repair. Although the mitochondria represent the major source of free radicals already under physiological conditions, other cell compartments such as microsomes and lysosomes participate in ROS production via oxidative reactions under stressful conditions. Increased activity of the cytochrome p450, particularly of the isoform Cyp2e1, has emerged as an important free radical generator during NASH [13]. Indeed, a direct association between Cyp2e1 and Cyp4a10 enzyme expression and the initiation of lipid peroxidation during the progression of NASH has been clearly identified [14]. Moreover, a critical role for NADPH oxidases (such as NOX2) in the development of features related to aggravation of NASH and, in general, of the metabolic syndrome has been convincingly demonstrated by Garcia-Ruiz et al. [15]. Following these considerations, many experimental and clinical studies based on scavenging or buffering of free radicals have begun to show promising results in a more relevant therapeutic context $[16,17]$. It is worth to mention that in the context of NASH several therapeutic options targeting oxidative stress via Nrf2 activation already achieved preclinical and clinical phase trials [18]. In particular, beyond classical natural antioxidant such as sulforaphane or resveratrol, the synthetic electrophilic compound oltipraz (dithiolethione) revealed promising effects in the treatment of liver metabolic diseases and is currently being analyzed in a phase II clinical trial.

The role of HGF/c-met axis in liver pathophysiology has been extensively investigated with a particular light on aspects regarding liver regeneration, hepatocyte proliferation, and apoptosis [19]. Moreover, activation of this signaling pathway has been repeatedly reported to exert hepatoprotective effects against experimental conditions characterized by oxidative stress $[20,21]$. In isolated hepatocytes, Clavijo-Cornejo et al. showed that HGF exerts a biphasic regulation of the NADPH oxidases with a shorttime effect inducing the activation of the enzyme and a long-time effect after which the persistence of a functional $\mathrm{HGF} / \mathrm{c}$-met pathway results to be necessary for the suppression of the NADPH oxidases components in an Nrf2dependent manner [22]. In line with these findings, a recent work from our group [5] demonstrated that c-met-deleted hepatocytes displayed enhanced oxidative stress and increased apoptotic cell death in association with overproduction of superoxide anion in vivo. This led to enhanced progression of hepatic inflammation and fibrosis. Recently, Dominguez-Perez et al. showed that administration of HGF reduces hepatocyte susceptibility to lipotoxicity through an increase of antioxidant defenses such as $\boldsymbol{\gamma}$-GCS and GSH thereby attenuating ROS formation and damage [23]. The generation of double mutant c-met/Keap $1^{\Delta \text { hepa }}$ mice further demonstrated that re-establishing a functional antioxidant activity completely reversed the accelerated pathological conditions observed in single c-met ${ }^{\Delta \text { hepa }}$ mice. In particular, the reduction of oxidative stress was accompanied by a decrease of the abovementioned pro-oxidant systems, Cyp2e1, Cyp4a10, and NOX2 expression. Conversely, expression of antioxidant cell weapons, such as catalase and thioredoxin-1, in addition to the well-known activation of the pentose phosphate pathway, was strongly upregulated. It is interesting to note that the amelioration of the redox balance occurred concomitantly with a reduced hepatic accumulation of triglycerides related to the inhibition of the LXR-dependent lipogenic program induced by Nrf2 as previously shown $[9,24]$. These findings actually consolidate our former data set illustrating reduced TG accumulation and oxidative stress in hepatocytes carrying a genetic activation of Nrf2 in two experimental models of diet-induced steatohepatitis [9]. Moreover, this aspect coherently matches with the activation of the AMPK/ PGC- $1 \alpha$ pathway observed in met/Keap $1^{\Delta \text { hepa }}$ hepatocytes. Although a causal relation with $\mathrm{Nrf} 2$ activation remains 


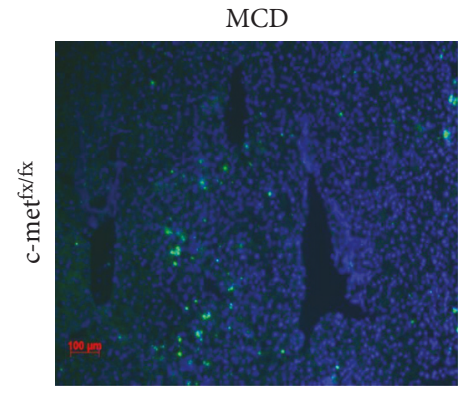

(a)

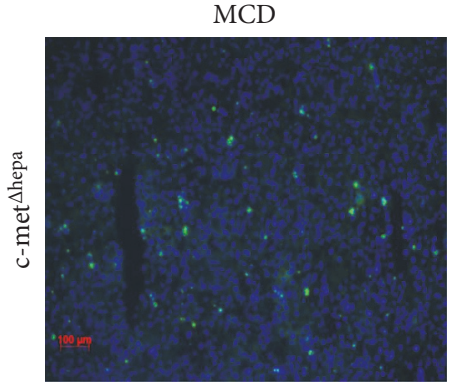

(b)

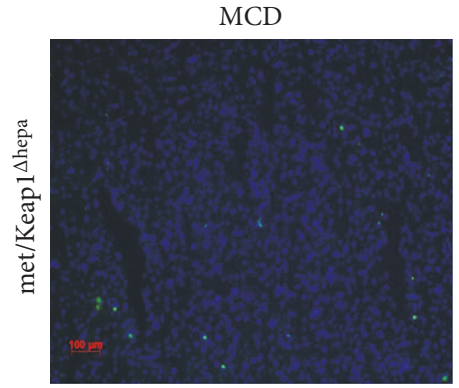

(c)

TUNEL

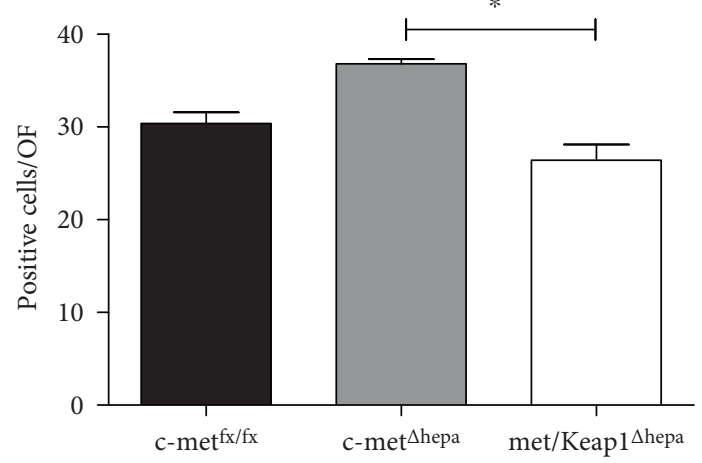

(d)

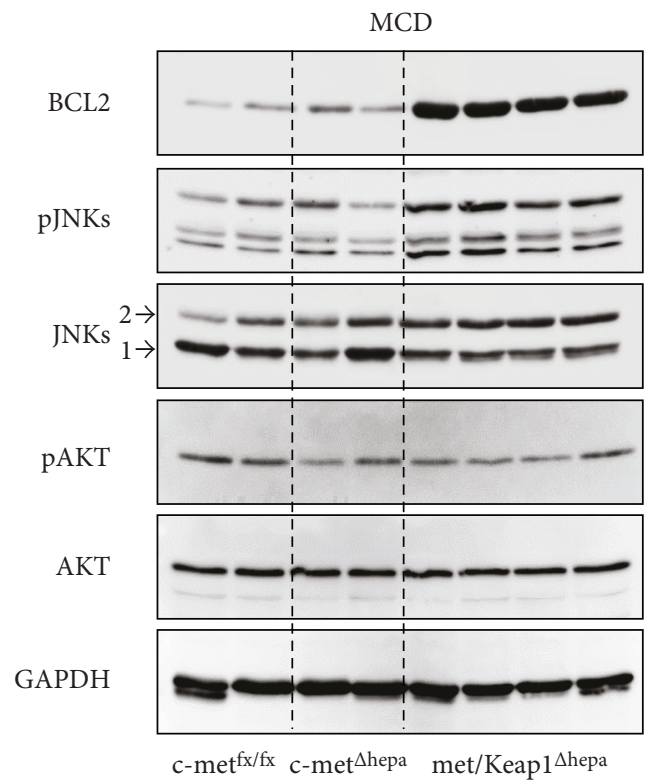

(e)

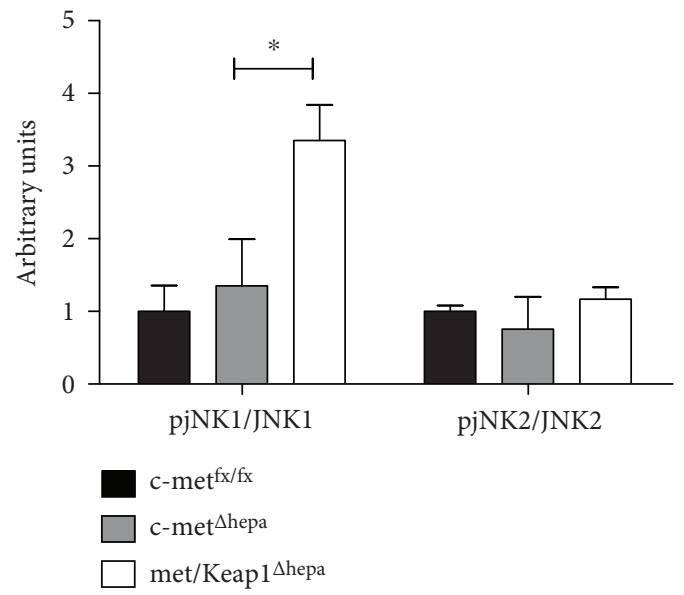

(f)

FIGURE 5: TUNEL immunofluorescence of $\mathrm{c}-\mathrm{met}^{\mathrm{fx} / \mathrm{fx}}(\mathrm{a}), \mathrm{c}-m e t^{\Delta \text { hepa }}(\mathrm{b})$, and met/Keap1 $1^{\Delta \text { hepa }}$ (c) liver sections (magnification $\left.100 \mathrm{x}\right)$ after 4 weeks of MCD feeding, with relative morphometric analysis (d). ${ }^{*}$ Data are expressed as mean \pm SE, ANOVA test with $p<0.05(N=5)$. Hepatic protein expression levels from Western blot (e) and densitometric analysis of band intensities (f) performed on total liver lysates of mice after $4 \mathrm{w}$ MCD administration.

poorly elucidated, Nrf2-dependent negative regulation of the lipogenic gene SCD-1 could contribute to hepatic AMPK activation as shown by Dobrzyn et al. [25]. Lastly, it would be reasonable to connect AMPK activation with maintenance of the NADPH production originating from the pentose phosphate pathway as an antioxidant mechanism in response to metabolic stress, as elegantly proposed elsewhere [26].

In relation to this observation, TUNEL-positive hepatocytes in double knockouts were strongly diminished as compared to those in control and single c-met knockouts. Interestingly, independent of the degree of steatosis, the loss 


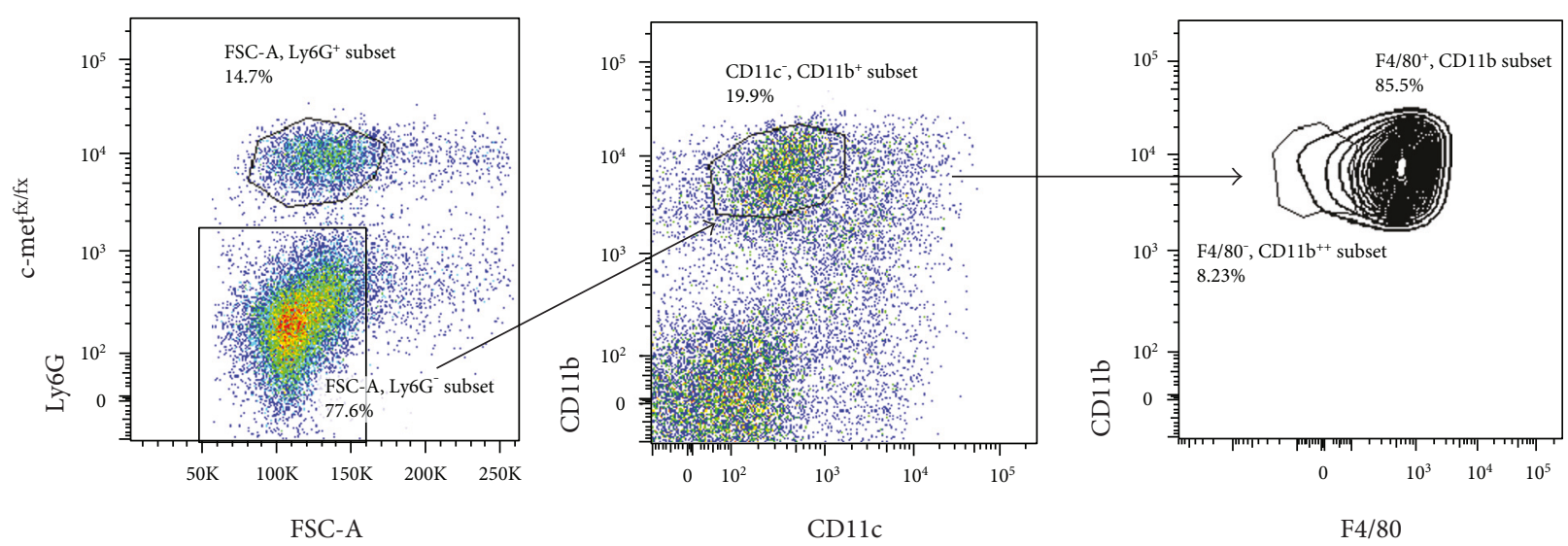

(a)
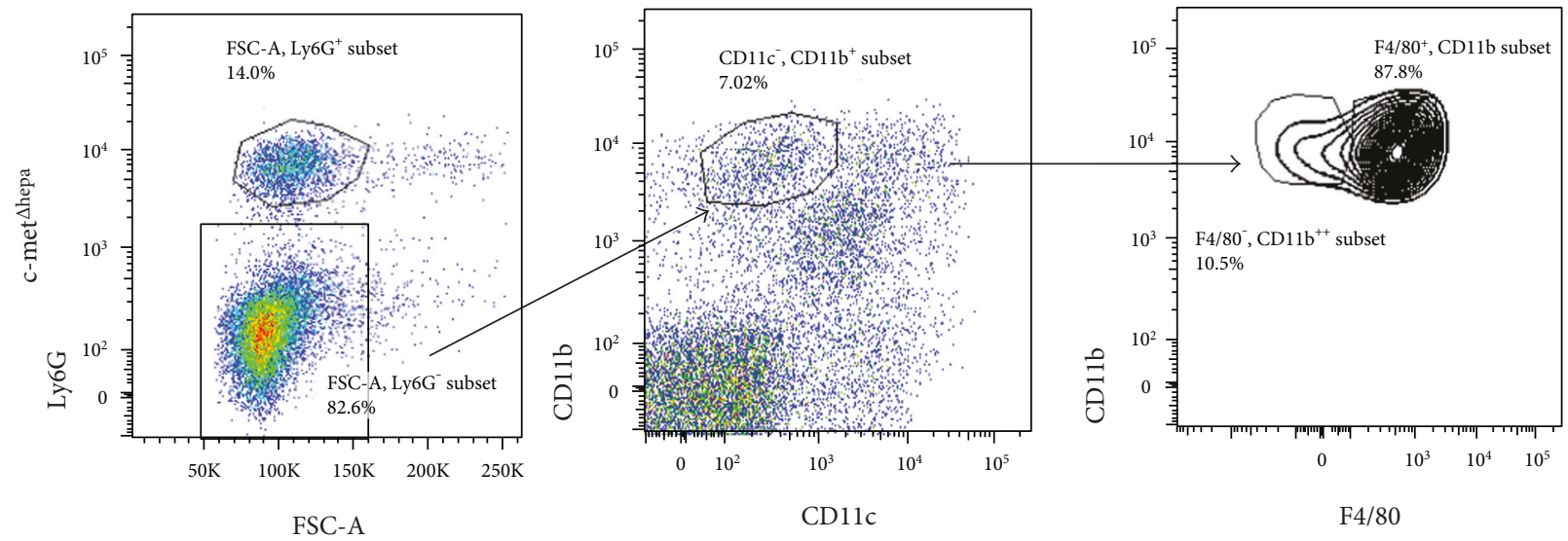

(b)
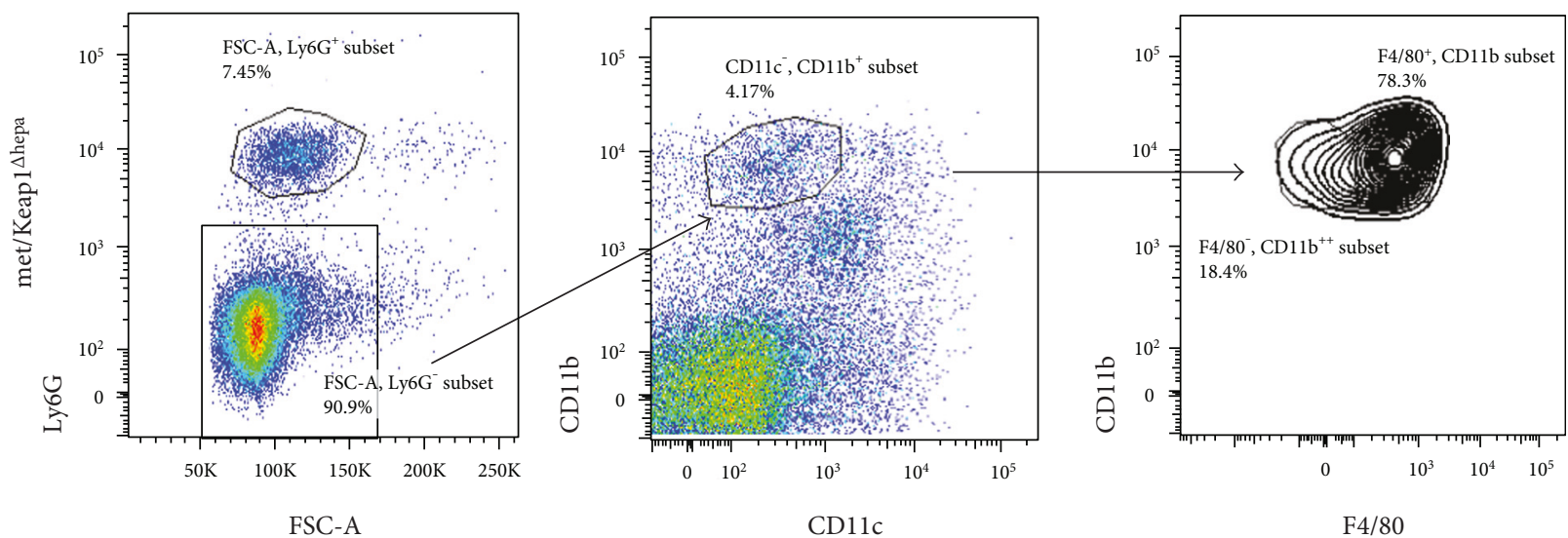

(c)

Figure 6: Continued. 


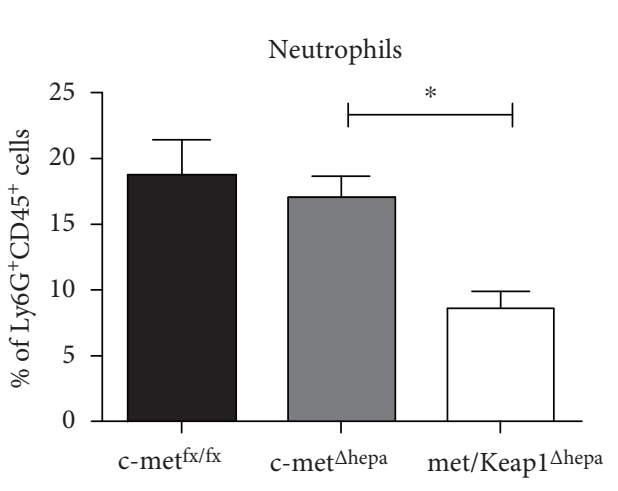

(d)

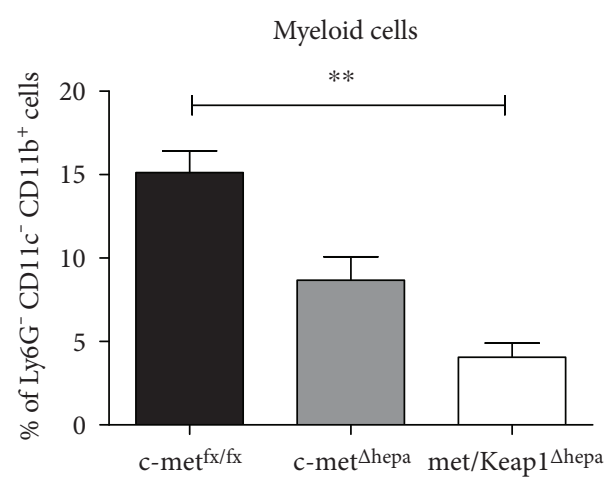

(e)

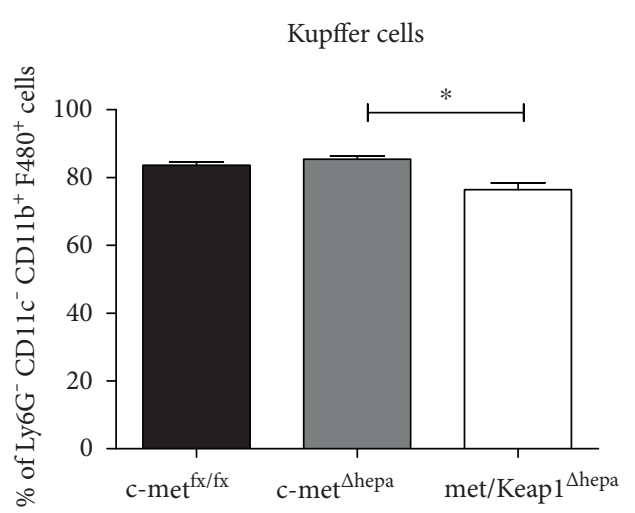

(f)

FIGURE 6: Representative gating strategy from flow cytometry analysis of intrahepatic neutrophils and monocyte/macrophage populations performed on total liver lysates of $\mathrm{c}-\mathrm{met}^{\mathrm{fx} / \mathrm{fx}}$ (a), c-met ${ }^{\Delta \text { hepa }}$ (b), and met/Keap1 $1^{\Delta \text { hepa }}$ (c) after 4 weeks of MCD feeding. Neutrophils

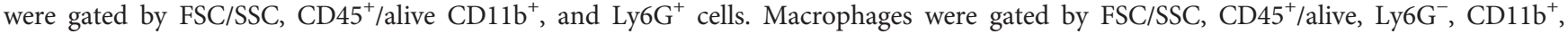
$\mathrm{F} 4 / 80^{+}$. Quantification of the gated populations reported on histogram (d, f). (e) ${ }^{* *} p$ values $<0.01$. ${ }^{*}$ Data are expressed as mean $\pm \mathrm{SE}$, ANOVA test with $p<0.05(N=3-5)$.

of c-met impairs hepatocyte capacity to counteract oxidative stress generated by fatty acid oxidation, thereby sensitizing cells to apoptosis. This suggests that reducing oxidative stress via Nrf2 activation might represent a key step in the protection from programmed cell death as we already observed in hepatocyte-specific Keap1 knockout mice [10]. Curiously, in the present work, cell protection was not associated with enhanced activation of the PI3K/AKT survival pathway. Thus, a significant increase of the stress-activated JNK phosphorylation was observed. Although the importance of this point results unclear, JNKs have been recently shown to promote nuclear Nrf2 activation [27]. Moreover, it might be relevant to underline that liver size was not significantly affected by administration of MCD diet in all the experimental groups and the double knockouts already display hepatomegaly under normal conditions. Analyses of immunofluorescence for the proliferation marker Ki67 indicated that hepatocyte proliferation seems to contribute only minimally to this phenotype. Thus, we reasonably believe that under normal chow conditions hepatomegaly might be the result of a synergistic effect of proliferation and cellular hypertrophy. Upon MCD feeding, a compensative proliferation in response to liver injury and hepatocyte cell death is commonly activated. Single hepatocyte-specific c-met deletion constantly results in diminished proliferation rate being partly responsible for the impaired tissue repair as previously reported. Genetic Nrf2 overactivation does not seem to have an impact on the proliferation rate but the extent of cell death is indeed dramatically reduced. Therefore, in conditions of metabolic stress, the increase in liver/body weight ratio observed in double knockouts results to be maintained through reduced cell death and increased cell functionality (hypertrophy).

In line with these findings, simultaneous deletion of cmet and Keap1 resulted in a dramatic decrease of infiltrating neutrophils $\left(\mathrm{Ly}_{6 \mathrm{G}}{ }^{+}\right)$and inflammatory monocytes $\left(\mathrm{CD}_{11 \mathrm{~b}}{ }^{+} /\right.$ $\left.\mathrm{F} 4 / 80^{+}\right)$. This indicates a modulation of the immune response possibly driven by reduced liver injury and through the negative modulation of specific inflammatory mediators. Although the reduction of neutrophils might be beneficial in the context of NASH in terms of a decreased MPO release [28], on the other hand, further studies are required in order to identify the phenotype of monocyte subpopulations since the cell polarization might differently influence the progression of the disease. In contrast, livers of $\mathrm{c}$-met/Keap $1^{\Delta \text { hepa }}$ mice display a significant increase of infiltrating $\mathrm{CD}^{+}$lymphocytes, characterized by a predominant $\mathrm{CD} 4^{+}$subtype. These findings seem to be consistent with a recent study showing that, in the context of $\mathrm{NASH}, \mathrm{CD}^{+}$lymphocytes 


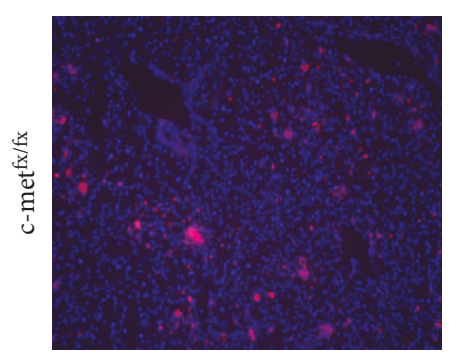

(a)

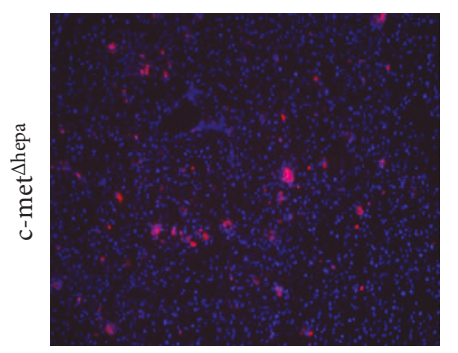

(b)

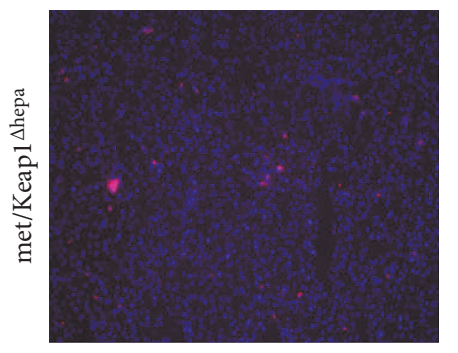

$\mathrm{CD} 11 \mathrm{~b}$

(c)

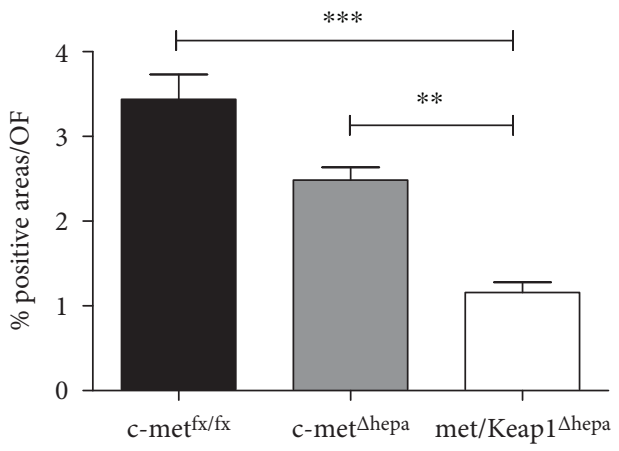

(g)

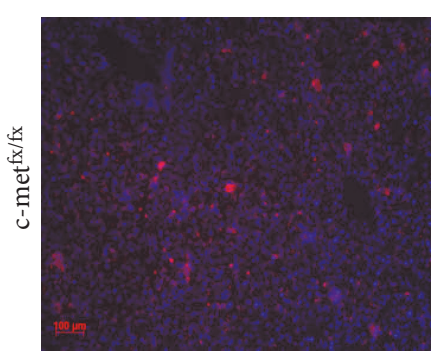

(d)

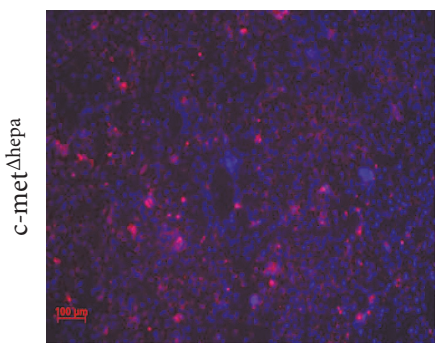

(e)

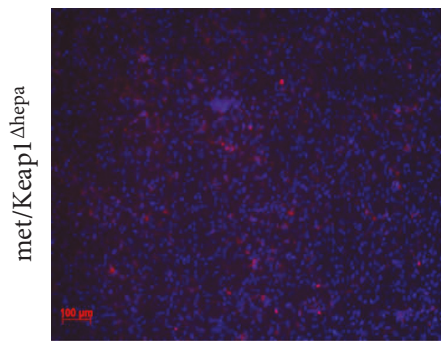

$\mathrm{F} 4 / 80$

(f)

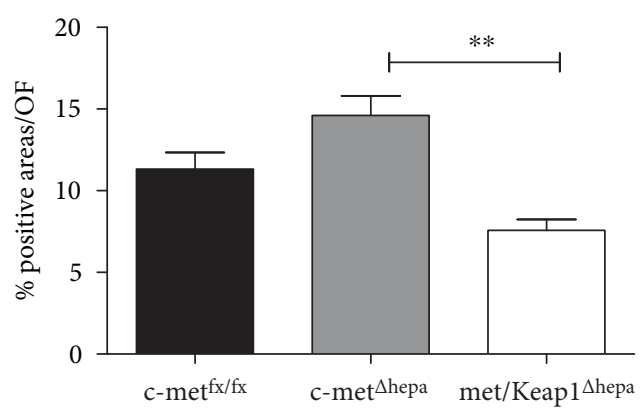

(h)

FIGURE 7: Immunofluorescence staining for neutrophil/monocyte marker CD11b (a, b, c) and macrophage marker F4/80 (d, e, f) of

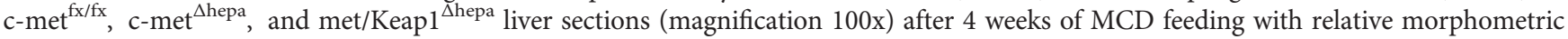
analyses $(\mathrm{g}){ }^{* * *} p$ values $<0.01$. (h) ${ }^{* *} p$ values $<0.01$. ${ }^{*}$ Data are expressed as mean \pm SE, ANOVA test with $p<0.05(N=3-5)$.

seem to be more susceptible to fatty acid-induced ROS cytotoxicity [29]. Consequently, a reduction of $\mathrm{CD} 4^{+}$population results to be deleterious in the progression of fibrosis. This aspect is going to emerge as a reproducible variable in several experimental conditions associated with hepatic Nrf2 overexpression. Further investigations are currently in progress in order to shed light on the nature and functions of this hepatic infiltrate.
Finally, loss of c-met in hepatocytes was previously shown to accelerate the onset of fibrosis not only in several experimental murine models [30] but also in NASH [5], through multiple mechanisms involving increased hepatocyte cell death, altered release of inflammatory mediators, oxidative stress, and cell proliferation. However, production of ROS remains a central player in the pathogenesis of liver fibrosis even if the causal relation in the context of cell 


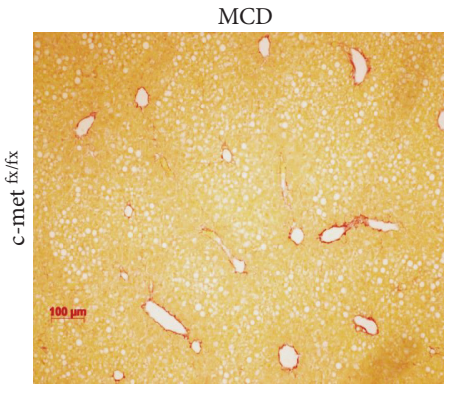

(a)

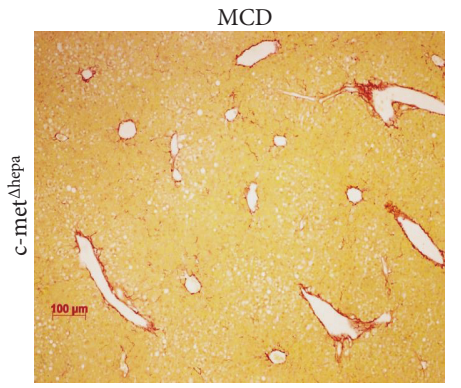

(b)

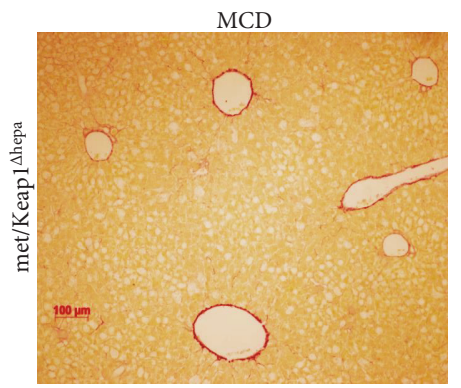

(c)

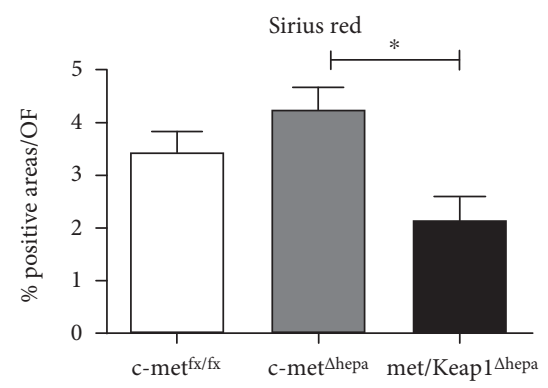

(d)

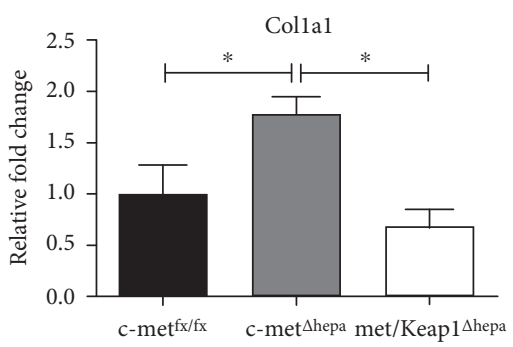

(f)

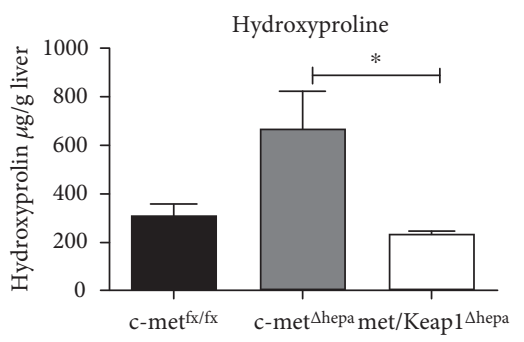

(e)

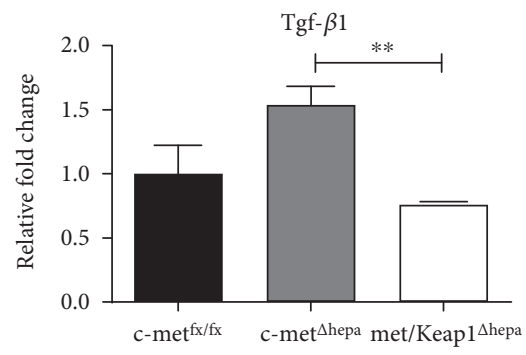

(g)

FIGURE 8: Sirius red staining of c-met ${ }^{\mathrm{fx} / \mathrm{fx}}(\mathrm{a}),{\mathrm{c}-\mathrm{met}^{\Delta \text { hepa }}}^{\mathrm{b}}$ ), and met/Keap1 ${ }^{\Delta \text { hepa }}$ (c) liver sections (magnification 100x) after 4 weeks of MCD feeding with relative morphometric analysis (d). Intrahepatic hydroxyproline content (e) and hepatic gene expression of profibrotic mediators $(\mathrm{f})(\mathrm{g}){ }^{* *} p$ values $<0.01$, from livers of mice after 4 weeks of MCD feeding. ${ }^{*}$ Data are expressed as mean \pm SE, ANOVA test with $p<0.05(N=5)$.

death and repair is still an argument of intense investigation [31]. Curiously, whereas overexpression of $\mathrm{Nrf} 2$ in c-met/Keap $1^{\Delta \text { hepa }}$ resulted in less $\mathrm{TG}$ accumulation, less oxidative stress, and reduced number of inflammatory cells as compared to controls and single knockouts, the degree of fibrosis in these mice was only moderately dampened to the degree of the control group. These data indicate that genetic enhancement of Nrf2 signaling is sufficient to repress MCD-dependent oxidative stress and cell damage but the efficacy against fibrosis seems to be restricted to a limited spectrum of transcriptional activation. To this end, identification of immune mediators directly regulated through Nrf2 transcriptional activity could shed light on the dark side of chronic overexpression of this transcription factor.

\section{Conclusion}

The results reported in this work offer further evidence for Nrf2-mediated cytoprotection (partly illustrated also in conference data set [32]). They pinpoint a key role for
$\mathrm{HGF} / \mathrm{c}-\mathrm{met}$ signaling in the regulation of redox homeostasis. Actually, genetic Nrf2 overexpression revealed therapeutic effects in c-met-deficient hepatocytes by counteracting oxidative stress thereby attenuating the disease progression. This work highlights critical aspects to be considered for the development of novel therapeutic strategies in the management of NASH.

\section{Conflicts of Interest}

The authors declare that there is no conflict of interest regarding the publication of this paper.

\section{Acknowledgments}

The authors would like to thank Dr. Daniel Heinrichs for the antibody concession. The work was funded by the Deutsche Forschungsgemeinschaft DFG: SFB/TRR57, Project P22. 


\section{References}

[1] M. Arechederra, R. Carmona, M. Gonzales-Nunez et al., "Met signaling in cardiomyocytes is required for normal cardiac function in adult mice," Biochimica et Biophysica Acta, vol. 1832, no. 12, pp. 2204-2215, 2013.

[2] M. Niimura, N. Takagi, K. Takagi et al., "Prevention of apoptosis-inducing factor translocation is a possible mechanism for protective effects of hepatocyte growth factor against neuronal cell death in the hippocampus after transient forebrain ischemia," Journal of Cerebral Blood Flow and Metabolism, vol. 26, no. 11, pp. 1354-1365, 2006.

[3] D. Ferraro, S. Corso, E. Fasano et al., "Pro-metastatic signaling by c-Met through RAC-1 and reactive oxygen species (ROS)," Oncogene, vol. 25, no. 26, pp. 3689-3698, 2006.

[4] R. Jagadeeswaran, S. Jagadeeswaran, V. P. Bindokas, and R. Salgia, "Activation of $\mathrm{HGF} / \mathrm{c}-\mathrm{Met}$ pathway contributes to the reactive oxygen species generation and motility of small cell lung cancer cells," American Journal of Physiology. Lung Cellular and Molecular Physiology, vol. 292, no. 6, pp. L1488-L1494, 2007.

[5] D. C. Kroy, F. Schumacher, P. Ramadori et al., "Hepatocyte specific deletion of c-Met leads to the development of severe non-alcoholic steatohepatitis in mice," Journal of Hepatology, vol. 61, no. 4, pp. 883-890, 2014.

[6] L. E. Gomez-Quiroz, D. Seo, and Y. H. Lee, "Loss of c-Met signaling sensitizes hepatocytes to lipotoxicity and induces cholestatic liver damage by aggravating oxidative stress," Toxicology, vol. 361-362, pp. 39-48, 2016.

[7] T. Suzuki and M. Yamamoto, "Molecular basis of the Keap1Nrf2 system," Free Radical Biology \& Medicine, vol. 88, Part B, pp. 93-100, 2015.

[8] O. Al-Sawaf, T. Clarner, A. Fragoulis et al., "Nrf2 in health and disease: current and future clinical implications," Clinical Science (London, England), vol. 129, no. 12, pp. 989999, 2015.

[9] P. Ramadori, H. Drescher, S. Erschfeld et al., "Hepatocytespecific Keap1 deletion reduces liver steatosis but not inflammation during non-alcoholic steatohepatitis development," Free Radical Biology \& Medicine, vol. 91, pp. 114-126, 2016.

[10] H. Okawa, H. Motohashi, A. Kobayashi, H. Aburatani, T. W. Kensler, and M. Yamamoto, "Hepatocyte-specific deletion of the keap1 gene activates Nrf2 and confers potent resistance against acute drug toxicity," Biochemical and Biophysical Research Communications, vol. 339, no. 1, pp. 79-88, 2006.

[11] M. Kaldenbach, A. Giebeler, D. F. Tschaharganeh et al., "Hepatocyte growth factor/c-Met signalling is important for the selection of transplanted hepatocytes," Gut, vol. 61, no. 8, pp. 1209-1218, 2012.

[12] M. L. Berres, R. R. Koenen, A. Rueland et al., "Antagonism of the chemokine Ccl5 ameliorates experimental liver fibrosis in mice," The Journal of Clinical Investigation, vol. 120, no. 11, pp. 4129-4440, 2010.

[13] M. A. Abelmegeed, A. Banerjee, S. H. Yoo, S. Jang, F. J. Gonzalez, and B. J. Song, "Critical role of cytochrome P450 2E1 (CYP2E1) in the development of high fat-induced nonalcoholic steatohepatitis," Journal of Hepatology, vol. 57, no. 4, pp. 860-866, 2012.

[14] I. A. Leclercq, G. C. Farrell, J. Field, D. R. Bell, F. J. Gonzalez, and G. R. Robertson, "CYP2E1 and CYP4A as microsomal catalysts of lipid peroxides in murine nonalcoholic steatohepatitis," The Journal of Clinical Investigation, vol. 105, no. 8, pp. 1067-1075, 2000.

[15] I. García-Ruiz, P. Solís-Muñoz, D. Fernández-Moreira, M. Grau, T. Muñoz-Yagüe, and J. A. Solís-Herruzo, "NADPH oxidase is implicated in the pathogenesis of oxidative phosphorylation dysfunction in mice fed a high-fat diet," Scientific Reports, vol. 6, p. 23664, 2016.

[16] S. Saphis, E. Delvin, J. M. Borys, and E. Levy, "Oxidative stress as a critical factor in nonalcoholic fatty liver disease pathogenesis," Antioxidants \& Redox Signaling, vol. 26, no. 10 , pp. 519-541, 2016.

[17] H. Perazzo and J. Dufour, "The therapeutic landscape of non-alcoholic steatohepatitis," Liver International, vol. 37, no. 5, pp. 634-647, 2017.

[18] G. Musso, M. Cassader, and R. Gambino, "Non-alcoholic steatohepatitis: emerging molecular targets and therapeutic strategies," Nature Reviews Drug Discovery, vol. 15, no. 4, pp. 249-274, 2016.

[19] S. S. Thorgeirsson, "The central role of the c-Met pathway in rebuilding the liver," Gut, vol. 61, no. 8, pp. 11051106, 2012.

[20] L. E. Gomez-Quiroz, V. M. Factor, P. Kaposi-Novak, C. Coulouarn, E. A. Conner, and S. S. Thorgeirsson, "Hepatocyte-specific c-Met deletion disrupts redox homeostasis and sensitizes to Fas-mediated apoptosis," The Journal of Biological Chemistry, vol. 283, no. 21, pp. 14581-14589, 2008.

[21] C. Enriquez-Cortina, M. Almonte-Becerril, D. Clavijo-Cornejo et al., "Hepatocyte growth factor protects against isoniazid/ rifampicin-induced oxidative liver damage," Toxicological Sciences, vol. 135, no. 1, pp. 26-36, 2013.

[22] D. Clavijo-Cornejo, C. Enriquez-Cortina, A. Lopez-Reyes et al., "Biphasic regulation of the NADPH oxidase by HGF/c-Met signaling pathway in primary mouse hepatocytes," Biochimie, vol. 95, no. 6, pp. 1177-1184, 2013.

[23] M. Dominguez-Perez, N. Nuno-Lambarri, D. Clavijo-Cornejo et al., "Hepatocyte growth factor reduces free cholesterolmediated lipotoxicity in primary hepatocytes by countering oxidative stress," Oxidative Medicine and Cellular Longevity, vol. 2016, Article ID 7960386, 8 pages, 2016.

[24] H. Y. Kay, W. D. Kim, S. J. Hwang et al., "Nrf2 inhibits LXR $\alpha$ dependent hepatic lipogenesis by competing with FXR for acetylase binding," Antioxidants \& Redox Signaling, vol. 15, no. 8, pp. 2135-2146, 2011.

[25] P. Dobrzyn, A. Dobrzyn, M. Miyazaki et al., "Stearoyl-CoA desaturase 1 deficiency increases fatty acid oxidation by activating AMP-activated protein kinase in liver," Proceedings of the National Academy of Sciences of the United States of America, vol. 101, no. 17, pp. 6409-6414, 2004.

[26] S. M. Jeon, N. S. Chandel, and N. Hay, “AMPK regulates NADPH homeostasis to promote tumour cell survival during energy stress," Nature, vol. 485, no. 7400, pp. 661-665, 2012.

[27] C. B. Ahn, J. Y. Je, Y. S. Kim, S. J. Park, and B. I. Kim, "Induction of Nrf2-mediated phase II detoxifying/antioxidant enzymes in vitro by chitosan-caffeic acid against hydrogen peroxide-induced hepatotoxicity through JNK/ ERK pathway," Molecular and Cellular Biochemistry, vol. 424, no. 1-2, pp. 79-86, 2017.

[28] B. Pulli, M. Ali, Y. Iwamoto et al., "Myeloperoxidase-hepatocyte-stellate cell cross talk promotes hepatocyte injury and fibrosis in experimental nonalcoholic steatohepatitis," Antioxidants \& Redox Signaling, vol. 23, no. 16, pp. 1255-1269, 2015. 
[29] C. Ma, A. H. Kesarvala, T. Eggert et al., "NAFLD causes selective $\mathrm{CD}^{+} \mathrm{T}$ lymphocyte loss and promotes hepatocarcinogenesis," Nature, vol. 531, no. 7593, pp. 253-257, 2016.

[30] A. Giebeler, M. V. Boekschoten, C. Klein et al., "c-Met confers protection against chronic liver tissue damage and fibrosis progression after bile duct ligation in mice," Gastroenterology, vol. 137, no. 1, pp. 297-308, 2009.

[31] N. J. Torok, "Dysregulation of redox pathways in liver fibrosis," American Journal of Physiology. Gastrointestinal and Liver Physiology, vol. 311, no. 4, pp. G667-G674, 2016.

[32] P. Ramadori, H. Drescher, S. Eschfeld et al., "Hepatic Nrf2 overexpression inhibits the deleterious effects induced by c-met deficiency in the progression of NASH," Zeitschrift für Gastroenterologie, vol. 54, no. 12, pp. 1343-1404, 2016. 


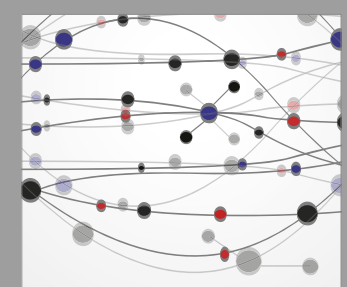

The Scientific World Journal
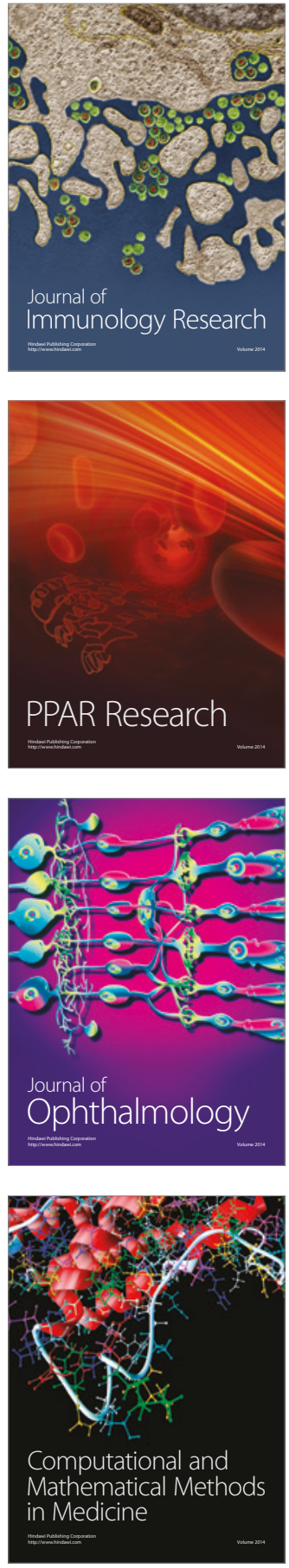

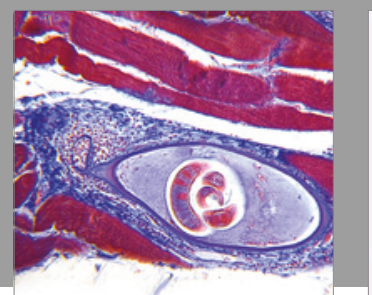

Gastroenterology Research and Practice
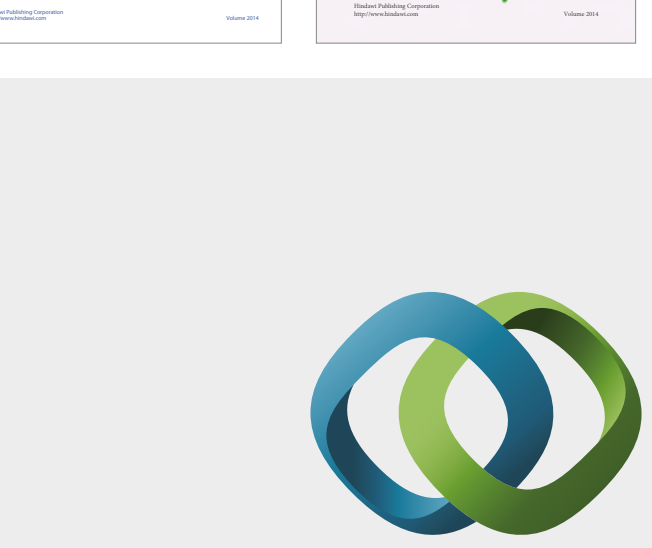

\section{Hindawi}

Submit your manuscripts at

https://www.hindawi.com
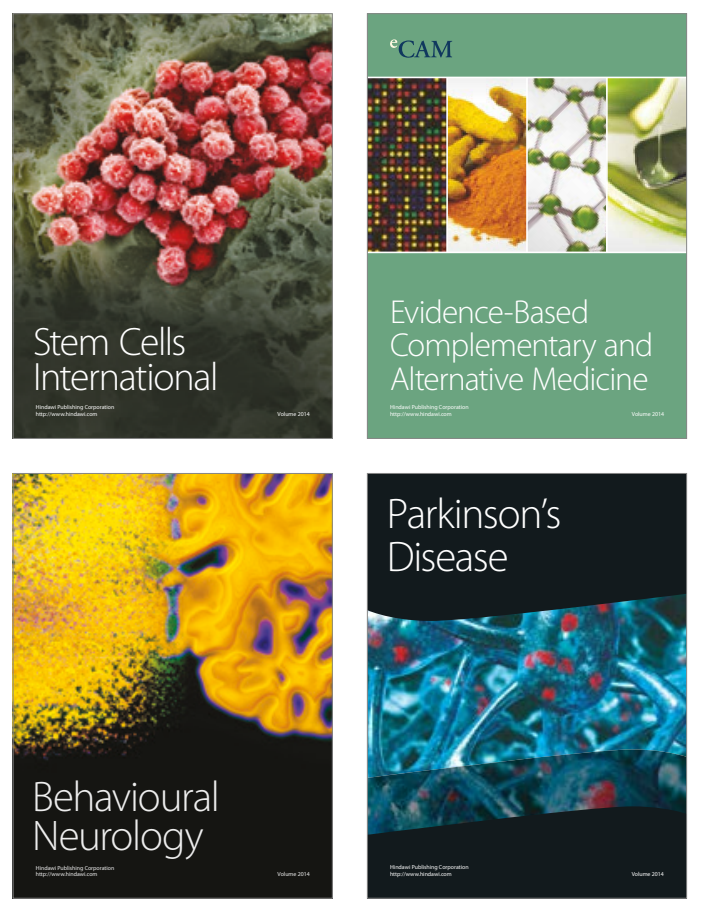
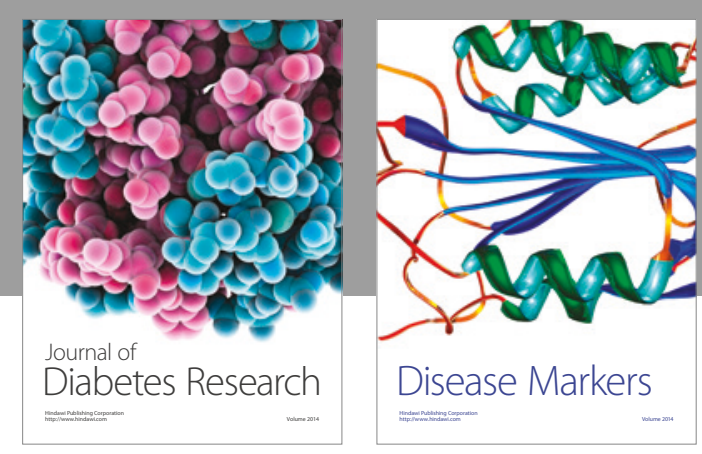

Disease Markers
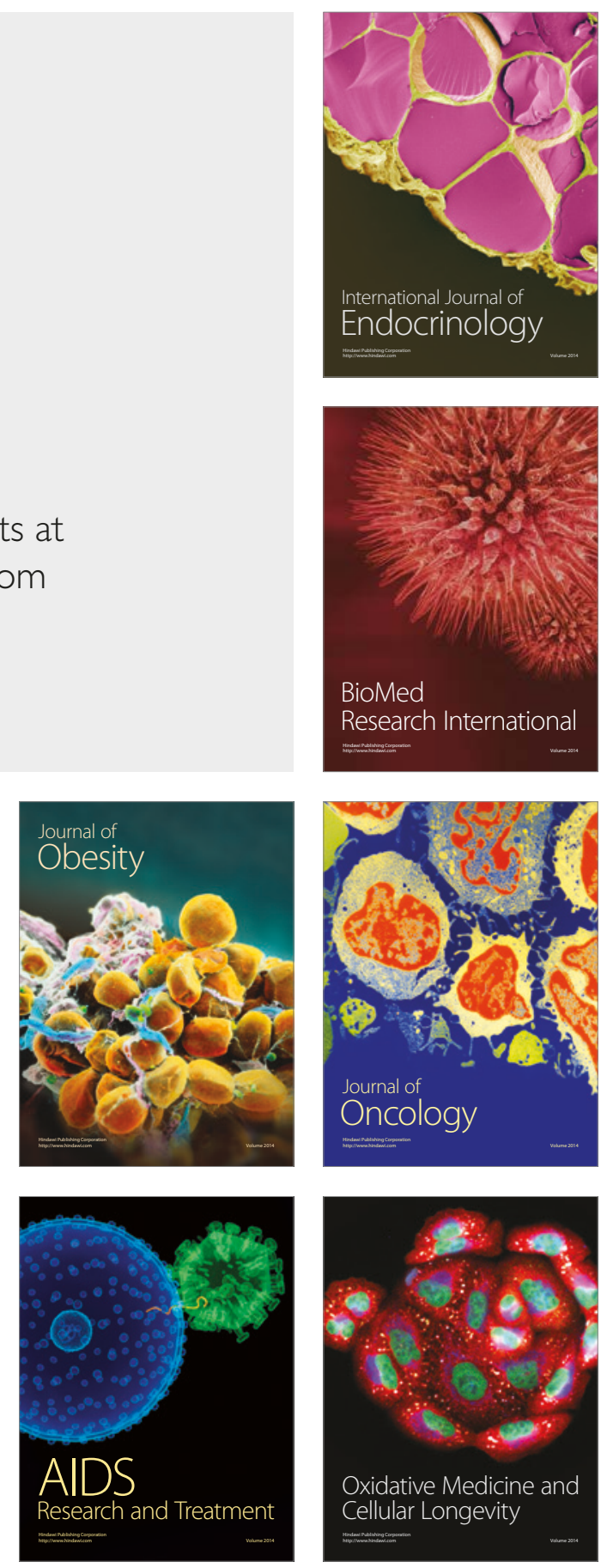